\title{
BIRDS
}

\section{0th ANNUAL SASKATCHEWAN CHRISTMAS BIRD COUNT - 2011}

\section{ALAN R. SMITH, Box 154, Avonlea, SK, SOH 0C0, Email: <alanrandi@sasktel.net>}

The Counts. For the second consecutive year the number of counts conducted increased over the previous year with 91 counts compared to 86 last year and 83 in 2009. No doubt the warm winter weather prompted more observers to venture out. Nine new or revived counts more than offset the four that were conducted last year but not this year.

The Weather. This past winter was memorable for its warm temperatures and meagre snowfall. The average daytime temperatures for all counts ranged from a low of -7 to a high of $-2^{\circ} \mathrm{C}$, while snow depth ranged from 4 to $13 \mathrm{~cm}$. Comparable figures for 2010 were -16 to $-11^{\circ} \mathrm{C}$, and 18 to $32 \mathrm{~cm}$. As we shall see, these unusual conditions had a profound effect on the numbers and distribution of birds on the $\mathrm{CBC}$.

The Birds. The 128,000 birds seen on the count were about average for the last 10 years, while the 102 species on count day was the highest since 2004 when 103 species were recorded.

Saskatoon with 48 species won for the $10^{\text {th }}$ consecutive year; Gardiner Dam and the Qu'Appelle Dam were both a distant second with 37 species. Although Estevan had only 35 count day species, it had an impressive 42 count period species.

Population Trends. The main beneficiaries of the warm weather were the waterbirds. Not only were new high counts set or tied for many species (Table 6), it is very likely that more species of waterbirds were on this count than any previous count. Thanks to the lack of snow, numbers of the two common field-feeding waterfowl, Mallard and Canada Goose, rebounded nicely from last year's lows and were higher than the five year average (Table 7).

Results for landbirds were markedly different. Upland gamebirds, Horned Lark, and Snow Bunting declined substantially (Table 7), and the Lapland Longspur was totally absent. These results are undoubtedly more apparent than real as there was no snow to force these species to roadsides to feed - they could forage anywhere!

Regina established a record high count for Snowy Owl with 45 birds (Table 6 ). Province-wide numbers were up a staggering $272 \%$ over the previous five years. The large number of young-ofthe-year observed leave little doubt that the species had a very good year on their Arctic breeding grounds. Their lemming prey must have been very abundant, and brood size of the owls must have been pushed to the limit!

The expansion of the Eurasian CollaredDove continues apace with a record high of 333 birds on 17 counts (plus 2 during the count period at Kyle). New localities included Cabri, Gardiner Dam, Outlook and Yorkton. In contrast, the expansion of the House Finch has leveled off with only two new localities (Balgonie and Shamrock) reporting the species.

Rarities. No new species were added 
to the all-time list of species seen on the CBC so the total remains at 184 species. Two species were, however, seen for the second time ever. These were a Winter Wren on the Fort Qu'Appelle count and an Orange-crowned Warbler on the Togo count. A Yellow-headed Blackbird on the Good Spirit Lake and two on the Indian Head count were the second and third records for the province. Also of note was our third record for Barrow's Goldeneye with one seen during the count period at Squaw Rapids. Northern Cardinals were seen at two localities: two during the count period at Prince Albert and one on count day at Yorkton.

Count areas and participants (Names of compilers are in italics).

1. ARCHERWILL. Gerald Hiron, Susan Hiron, Audrey Hnetka, Elaine Hughes, Dorothy Klettberg, Judy Revoy.

2. AVONLEA. Alan Smith, Blaine Sudom.

3. BALGONIE. Keith Barr, Jared Clarke, Laurie Koepke, Kim Mann, Val Mann, Kristen Martin, Moira McKinnon, Brett Quiring, Jordan Tholl, Jenna Tokaruk, Matthew Tokaruk, Duncan Willis

4. BANGOR. Allan Bolton, Lynn Bolton, Aileen Hayward, Alan Hayward, John Maddaford, Leslie Maddaford, Bev McLaren, Raye McLaren.

5. BIGGAR. Murray Newton, Mark Pickett, Linda Schnedar, Lorrie Sielski, Larry Sutherland, Rita Sutherland, Guy Wapple, Marguerite Wapple, Rob Wapple.

6. BIRCH HILLS. Doug Boivin, Carman Dodge, Margaret Mareschal, Maurice Mareschal, Don Weidl.

7. BROADVIEW. Doug Boivin, Barbara Weidl, Don Weidl.
8. CABRI. Carman Dodge, Ron Jensen, Don Weidl.

9. CANDLE LAKE. Carman Dodge, Margaret Mareschal, Maurice Mareschal, Don Weidl.

10. CATER. Beverly Beland, Orval Beland, Donna Long, Waldy Long.

11. CHATSWORTH S.D. George Murray, Laurie Murray, Herb Rooke, Stewart Rooke, Carol Tangedal, Torrie Tangedal.

12. CHRISTOPHER LAKE. Jeannie Walker.

13. CHURCHBRIDGE. Cas Chyz, Verna Chyz, Elin Johnson, Ron Johnson, Phyllis Kaeding, Martha Karau, Wally Karau, Elaine Pollock, John Pollock, Denis Putland, Dianne Putland, Ed Wirth, Gloria Wirth.

14. CLARK'S CROSSING. Carol Blenkin, Lucille Bradatsch, Annemarie BuchmannGerber, Dave Cook, Louise Cook, Lorne Duczek, Terry Hams, Marilyn Haskins, Richard Hedley, Robert Johanson, Richard Kerbes, Henry Klypak, Gordon Koshinsky, Margaret Koshinsky, Audrey MacKenzie, Bill MacKenzie, Hilda Noton, Sylvia Raginski, Jan Shadick, Stan Shadick, Marten Stoffel, Mary Toews, Guy Wapple, Michael Williams.

15. CODETTE LAKE. Doug Boivin, Barb Weidl.

16. CORONACH. Ryan Dudragne, Dan Sawatzky, Alan Smith.

17. CRAVEN. Barbara Barnett, Lyn Brokofsky, Tina Campbell, Stephane Canevet, Jim Cummings, Amanda Douglas, Ryan Dudragne, Karen Eliot, Ruth Englund, Elaine Exelby, Gabriel Foley, Louise Holloway, Phil Holloway, Fran Kerbs, Laurie Koepke, Bob 
Luterbach, Kent Lynn, Barbara Mader, Doug Mader, Lauren Mang, Kim Mann, Rocky Marchigiano, Jaret McDonald, Barry Mitschke, Valerie Mitschke, Kevin Moore, Jeff Nye, Shelley Nye, Mary Lynn Phillips, Curtis Pollock, Brett Quiring, Al Sumla, Jeannette Taylor, Robb Taylor, Mary Weber.

18. CREIGHTON. Brenda Schmidt, Harvey Schmidt.

19. CROOKED LAKE. Boyd Metzler.

20. CROOKED RIVER. Helen Carson, Lyle Carson, Burnice Lewis, Joyce Lewis, Margaret Mehler.

21. CYPRESS HILLS PROVINCIAL PARK (Centre Block). David Larson, Margarette Larson, Brynne Martin, Mimi Martin, Melody Nagel-Hisey, John Schock.

22. DENHOLM. Beverly Beland, Gerard Beland, Orval Beland.

23. DORINTOSH. Joe Twidale, Lorraine Twidale.

24. EASTEND. Roxie Binkley, Duane Bristow, Kevin Bristow, Robert Gebhardt, John Hanlin, June Higgins, Harvey Johnson, Keith Schwett.

25. ENDEAVOUR. Norman Harris.

26. ESTEVAN. Batty Dies, Marilyn Dies, Kathy Hedegard, Rose Walton, Guy Wapple.

27. ESTUARY NORTH. Cathy Cocks, Dean Francis.

28. FENTON. Carman Dodge, Maurice Mareschal, Don Weidl.

29. FORT QU'APPELLE. Jim Armstrong, Mark Gerema, Peter heerschop. Shiela
Heerschop, Alice Isfan, Jack Lowe, Jean McKenna, Alan Mlazgar, Vivian Morcom, Paul Paquin, Wendy Paquin, Brett Quiring, Janaye Starr, Keith Stevens, Kim Stevens, Ted Stevenson, Colette Stushnoff, Richard Stushnoff, David Sutherland, Rose Vernoy, Fred Warren, Bill Wendell.

30. GARDINER DAM. Ryan Dudragne, Greg Fenty, Maxine Forsberg, Jeff Jensen, Ron Jensen, Nick Saunders, Marten Stoffel, Guy Wapple.

31. GOOD SPIRIT LAKE. Bill Anaka, Dorothy Riesz, Ray Riesz, Lloyd Wilson, Ray Wilson.

32. GRANDORA. Danelle Messer, Mika Messer, Verna Messer.

33. GRASSLANDS NATIONAL PARK. Krista Bekker, Nick Cairns, Ervin Carlier, Eugene Dumont, Pat Fargey, Michael Fitzsimmons, Johane Janelle, Matthew Johnson, Kevin Moore, Wes Olson, Katherine Patterson, Taylor Roy, Colin Schmidt, Robert Sissons, Karin SmithFargey, Ashley Wruth.

34. GRAYSON. Carina Helm, Charles Helm, Daniel Helm, Linda Helm, Jeanette Zimmer, Karl Zimmer.

35. HARRIS. Nick Saunders, Guy Wapple.

36. HAZLET. Laurent Dudragne, Ryan Dudragne.

37. HUDSON BAY. Gloria Allchin, John Daisley, Ian Daisley, Joyce Hayes, Les Hayes, Agnes Lewellin, Peter Lewellin, Dennis Reimer, Ruth Reimer, Lee Smith, Delia Smith, Gloria Stang.

38. INDIAN HEAD. Jan Beatty, Mike Duran, Eileen Escott, Irv Escott, Mavis Jealous, Jim Jinks, Linda Jinks, Roy 
Hearn, Nick Kucharan, D. Loran, Dora Nichols, Adam Scott, Brian Scott, Bob Scott, Glenn Scott, Lorne Scott, Chad Skinner, Christine Skinner, Fred Skinner, Donna Tompson.

39. KENASTON. Doug Beckie, Drew Beckie, P. Lawrence Beckie, Tyler Beckie.

40. KENOSEE LAKE. Boyd Metzler, John Pollock.

41. KETCHEN NORTH. Dallas Fairburn.

42. KILWINNING. Ed Driver, Gerry Threlfall.

43. KINDERSLEY NORTH. Jean Harris, Keith Harris.

44. KINLOCH. Don Forbes, Doreen Forbes, Cliff Logan, Joyce Logan, Doreen Wickstrom.

45. KYLE. Greg Nelson, Glen Pederson, Ed Stepple, Dan Zazelenchuk.

46. LAC LAPLONGE. Katherine Brewster, Erin Conkin, John Conkin, Aislinn Petz.

47. LA RONGE. John Schisler.

48. LAST MOUNTAIN LAKE N.W.A. Valeriana Harris, Shiela Lamont.

49. LAST MOUNTAIN LAKE N.W.A.NOKOMIS. Ross Dickson, Lois Vanthuyne.

50. LOVE - TORCH RIVER. Carol Blenkin, Hal Birkett, Joan Birkett, Bert Dalziel, Duke Dalziel, Joan Dalziel, Sara Dalziel, Jack Dewar, Lena Dewar, Scott Edwards, Ashley Gallon, Roseanne Kirkpatrick, Ron Knutson, Terry Knutson, Eilene L'Heureux, Jennette LeCuyer, Kyla LeCuyer, Bev Macauley, Dave Macauley, Lillian Nesset, Beatrice Runn, Jeanette
Runn, John Sinclair, Joshua Turtle, Leonard Turtle.

51. LUSELAND. Kim Finley, Estelle Finley, Graeme Finley, Liam Finley, Valerie Finley, Brent Honeker, Shirley Honeker.

52. MACDOWALL. Hazel Barton, Myron Barton, Dianne Brown, Ron Brown, Barb Sather, Alan Smith, Ione Surbey, Bob Tunstall.

53. MACNUTT. John Skinner, Tim Wendell.

54. MATADOR. Jim Romo, Cindy Romo.

55. MEADOW LAKE. Bill Caldwell, Janet Caldwell, Marlynne Sampson, Monty Sampson, Bob Wilson, Ian Wilson.

56. MOOSE JAW. Ryan Dudragne, Jeff Mander, Alan Smith.

57. MOOSE MOUNTAIN. Bob Cameron, Kathy Hedegard, Doyle Thomas, Val Thomas.

58. MORSE. Jared Clarke, Noel Enns, Stella Enns, Mike Francis, Kristen Martin, Randy McCulloch, Pete McHugh, Joel Priebe, Ken Priebe, Myrna Priebe, Lori Wilson.

59. NIPAWIN. Carol Blenkin, Vi Budd, Joyce Christiansen, Bert Dalziel, Carman Dodge, Rick Douslin, Patti Gaertner, Joe Graumans, Jennette LeCuyer, Doug Phillips, Sherley Phillips, Leonard Turtle, Barb Weidl, Don Weidl.

\section{NISBET FOREST, NORTHWEST.}

Sandra Jewell, Beth Neil, Dale Neil.

\section{NISBET FOREST, WEST.}

Kim Clark, Kiri Clark, Shamara Clark, Suzanne Clark. 
62. ODESSA. Arden Curts, Denise Curts, Denny Curts.

63. OUTLOOK. Alan Smith, Graham Thomson.

64. PIERCE LAKE. Bill Bretsch, Ted Hindmarsh, Richard Klauke, Phil Shore.

65. PIKE LAKE. Alison Baudru, Lawrence Beckie, Carol Blenkin, Katherine Brewster, Ann Christensen, David Cook, Louise Cook, John Conkin, Jill Cornford, Peter Cornford, Yvonne Cuttle, Elsbeth Dormuts, Betty Ann Dunlap, Lorne Duczek, Kelly Fairbrother, Joe Fry, Kathy Fry, Bob Girvan, Michael Gollop, Bob Green, May Haga, Greg Hutchings, Ron Jensen, Robert Johanson, Bob McNaughton, Hilda Noton, Lynn Oliver, Scotty Oliver, Keith Pahl, John Patterson, Sylvia Raginski, Frank Roy, Marc Sabourin, Nick Saunders, Jan Shadick, Stan Shadick, Linda Schnedar, Marten Stoffel, Joe Stookey, Louisa Stuglin, Stephan Stuglin, Bev Will, Michael Williams, Scott Wilson, Jim Wood, Dwight Young.

66. PRINCE ALBERT. Ralph Abrey, Danika Benson, Doug Braaten, Marie Braaten, Kim Clark, Shamara Clark, Carman Dodge, Hamilton Greenwood, Ruth Griffiths, Bencky Grimwood, Kaylee Hoko, Sandra Jewell, Gwen Klebek, Jamie Krip, Brenda Lee, Justine Lofstrom, Randell Lofstrom, Joanne Marchand, Elaine McMillan, Alan Porter, Francine Prins, John Rye, Bonny Tolley, Don Weidl, Marvis Willis.

67. PRINCE ALBERT N.P. Shannon Bond, Kayla Brunner, Seth Cherry, Carlene Gorecki, Digit Guedo, Dusty Guedo, Courtney Jansen, Jonathan Jansen, Eric Knight, Lance Langford, Jacki Leason, Darcy Lebel, Brad Lloyd, Heather MacPhee, Fiona Moreland, Joanne Reimer, Gregg Rutten, Terry
Schneider, Theresa Stene, Norman Stolle, Rod Thompson, Coralee Vaillancourt, Jeff Weir, Dave Weider.

68. QU'APPELLE. Christine Blair, Frank Veresh.

69. QU'APPELLE VALLEY DAM. Ryan Dudragne, May Haga, Richard Hedley, Robert Johanson, Nick Saunders, Jan Shadick, Stan Shadick Marten Stoffel, Guy Wapple, Michael Williams.

70. RAYMORE. Valeriana Harris, Shiela Lamont.

71. REGINA. Keith Barr, Lionel Bonneville, Stephane Bonneville, Lola Mae Crawley, Ron Crawley, Jim Cummings, Kathleen Donhauer, Jim Elliot, Ruth Englund, Terry Ford, Shirley Friel, Kathy Hamre, Dale Hjertaas, Myrna Hunter, Phyllis IIsley, Rhéal Larouche, Lucille Lipka, Mike Lipka, Bob Luterbach, Lauren Mang, Kim Mann, Val Mann, Moira McKinnon, Kevin Moore, Dorothy Nordquist, Jim Nordquist, Catherine Parkinson, Wayne Pepper, Brett Quiring, Dan Sawatzky, Evanna Simpson, Margaret Skeel, Frank Switzer, Steven Weir, Linda Winkler.

72. ROKEBY. Lloyd Liebracht, Lorn Niebergal, Fred Phillips, Martin Phillips, Kirk Scrernick, Ray Thies.

73. ROSCOMMON S.D. Kate Althouse, Marjorie Finnie, Ruby Finnie, Brian Irving, Allen O'Brien, Dianne Sloan, Graham Sloan, Marguerite Sloan.

74. ROULEAU. Lucas Babcock, Allen McGratten, Noreen McGratten, Patricia Sterzuk.

75. ROUND LAKE (Qu'Appelle Valley). Boyd Metzler, Mary Ward, Pat Ward.

76. SALTCOATS. Arden Bradford, Donna Bradford, Olga Brygadier, Walter 
Brygadier, Gregor Farquharson, Joan Farquharson, Kaito Farquharson, Walter Farquharson, James Jowsey, Shirley Jowsey, Betty Michael, Herb Rooke, Stuart Rooke, Gloria Rathgeber, Valerie Trowell, Joan Wilson, Rob Wilson.

\section{SASKATCHEWAN LANDING} PROVINCIAL PARK. Dawn Odegard, Glen Pederson, Mike Redmond, Marten Stoffel, Dan Zazelenchuk.

78. SASKATCHEWAN RIVER FORKS. Carman Dodge, Ron Jensen, Nick Saunders, Don Weidl.

79. SASKATOON. Tony Allen, Alison Baudru, Lou Baudru, Neva Bayliss, Janny Bos, Heather Brenneman, Heinz Buchman, Annemarie Buchmann-Gerber, Mike Chorney, George Christenson, Jacquie Christenson, Dave Cook, Louise Cook, Ken Coutu, Ann Coxworth, Ewen Coxworth, Yvonne Cuttle, Bill Davenport, Dean Davenport, Sonia Davenport, Ron Delver, Lorna Doerksen, Lorne Duczek, Melanie Elliott, Tammy Elliot, Kelly Fairbrother, Bob Ferguson, Joanne Ferguson, David Forbes, Cathy Fry, Joe Fry, Kamal Gabadage, Daniel Giesbrecht, Mike Gollop, Bob Green, Rita Gruodyte, May Haga, Audrey Hall, Terry Hams, Peter Hardie, Brenda Harris, Donna Haugen, Andrea Hull, Diana Hull, Kathryn Hull, Peter Hull, Ron Jensen, Marlene Kalanack, Arlene Karpan, Robin Karpan, Karla Lavery, John Lavery, Audrey MacKenzie, Bill MacKenzie, Bob McNaughton, Colleen Meldrum, Larry Mitchell, Scott Mitchell, Hilda Noton, John Patterson, Asha Perera, Alison Philips, Dorothy Reimer, Paul Reimer, Jordanna Roesler, Marella Rosta, Marc Sabourin, Craig Salisbury, Trish Santo, Scott Saretzky, Nick Saunders, Marjorie Scharf, Murray Scharf, Doug Schmeiser, Irene Schmeiser, Linda Schnedar, Eileen Shpyth, Henry Shpyth, Laurie Slinger, Jan Solem, Penny Stalker, Marten Stoffel,
Mary Toews, Hilda Voth, Guy Wapple, George West, Bev Will, Michael Williams, Amy Wilson, Scott Wilson, Jim Wood, Sandy Woynarski, Stan Woynarski, Norman Zlotkin.

80. SAWYER LAKE. Maureen Blight, Kathleen Pitt, Michael Pitt, David Weiman.

81. SHAMROCK. Mike Francis, Hugh Henry, Milton Knudsen, Sylvia Knudsen, Joel Priebe, Myrna Priebe, Lori Wilson.

82. SNOWDEN. Sylvia Debbler, Sonja Fidyk, Diane Friesen, Ed Hagel, Irene Hagel, Wayne McAuley, Linda Patton, Jack Pickett, Heather Priestley, Karen Priestley, Valerie Rein, Bill Thompson, Tim Thompson, Irene White.

\section{SPINNEY HILL. Ed Driver, Margaret} Driver.

\section{SQUAW RAPIDS. Ryan Dudragne,} Dan Sawtzky.

85. SWIFT CURRENT. Jim Beattie, Jacquie Bolton, Stacy Bolton, Laurent Dudragne, Mary Ann Dudragne, Ryan Dudragne, Dave Green, Esther Green, Katie Hagman, Norma Hain, Leonard Head, Hugh Henry, Leonard Howes, Lois Howes, Witt Howes, Stephanie Kaduck, Janet Payne, Harold Steppuhn, Irene Stinson, Kae Waters, Tom Waters.

86. TOGO. Kevin Chutskoff, Donna Dewores, Barb Elsasser, Doug Elsasser, Amanda Harper, Anita Huziak, Ed King, Louise King, William Koreliuk, Karen Leis.

87. WEYBURN. Cecil Burr, John Ferrier, Norm Flaten, Millie Fleming, Glen Fleming, Cyril Marcotte, Elma McCormick Charlotte Payak, Don Payak, Doyle Thomas, Val Thomas, Kim Thorson, Myrt Thorson, John Whitell.

88. WHITE BEAR. Dan Zazelenchuk, Sig Jordheim. 
89. WHITEWOOD. Cindy Ashfield, Joe Ashfield, Paul Ashfield, Freda Ede, Tom Ede, Art Hinze, Joyce Kydd, Florence Luhtala, Sarah Mambourg, lla Meszaros, Boyd Metzler, Harry Mitchell, Marilyn Mitchell, Donna Mohr, John Pollock, Dawn Vennard, Diane Veresh, Pat Ward.

90.WINGARD-FORT CARLTON. Myron Barton, Rebecca Beam, Laurel Jensen, Ruth Prosser, Alan Smith, Robert Zerebeski.
91. YORKTON. Bill Anaka, Bill Beck, Vern Brown, Jim Dicker, Pat Fletcher, Cheryl Fraser, Joyce Gardner, Bob Graham, Lorraine Harris, Lance Irvine, Ethel Krekiewetz, Sheldon Knoll, Joanne Loveridge, Joyce Muir, Kaarina Rahn, Gloria Rathgeber, Dorothy Riesz, Ray Riesz, Bonnie Rushowick, Geoff Rushowick, Patrick Rushowick Dale Sharpe, Gary Shury, Dorothy Skene, Stan Williams, John Young.
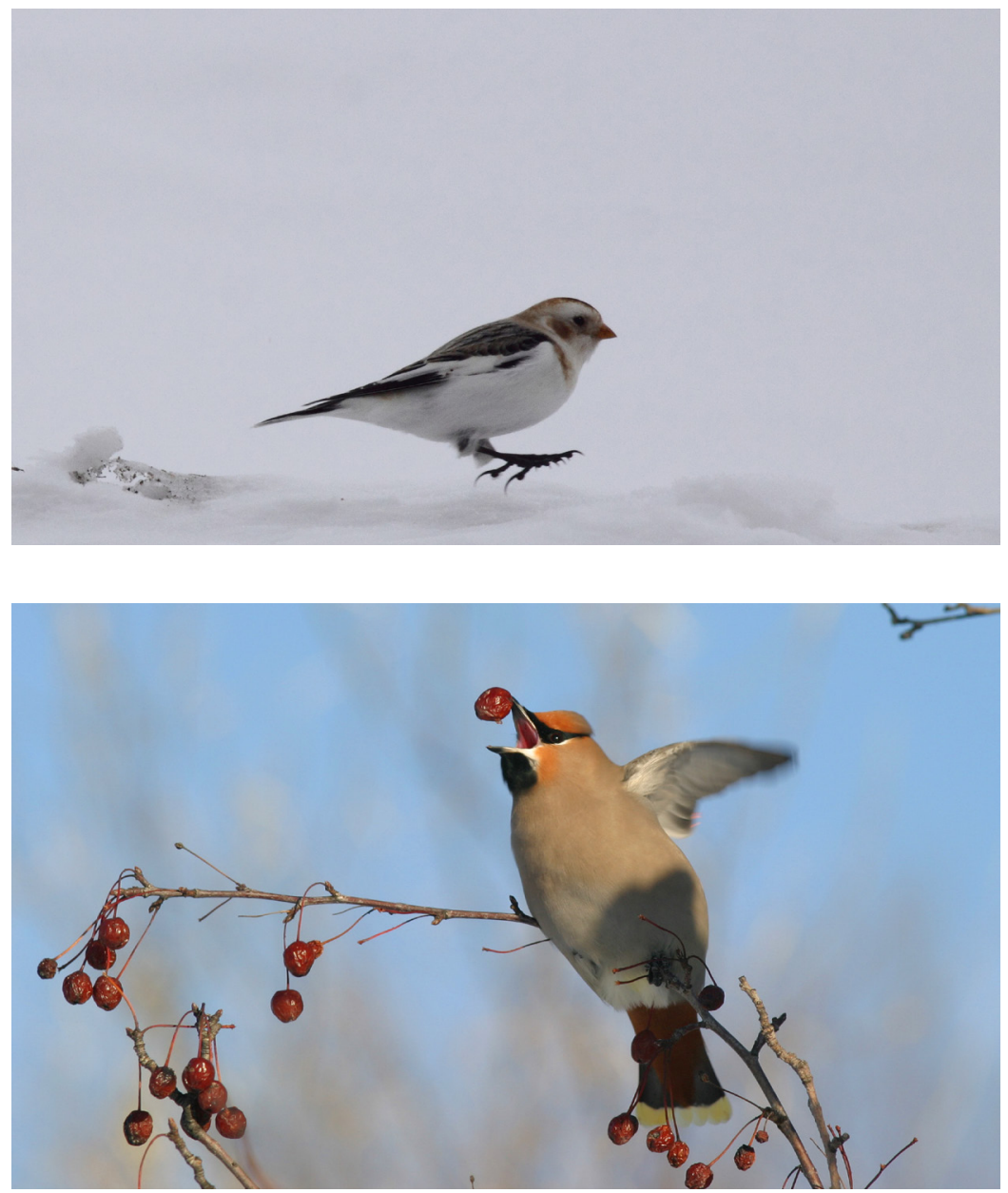

Top - Snow Bunting

Bottom - Bohemian Waxwing

C. Artuso 


\begin{tabular}{|c|c|c|c|c|c|c|c|c|c|}
\hline Locality & Date & 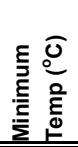 & 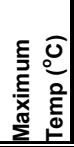 & 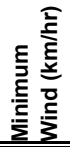 & 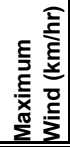 & 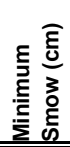 & 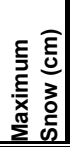 & Sky A.M. & Sky P.M. \\
\hline Archerwill & 17 Dec 2011 & -11 & -5 & 5 & 5 & 10 & 10 & partly cloudy & partly cloudy \\
\hline Avonlea & 4 Jan 2012 & 3 & 11 & 0 & 0 & 0 & 0 & clear & clear \\
\hline Balgonie & 2 Jan 2012 & -21 & -5 & 30 & 45 & 0 & 10 & mostly clear & mostly clear \\
\hline Bangor & $28 \operatorname{Dec} 2011$ & -10 & -1 & 5 & 10 & 4 & 10 & mostly clear & partly cloudy \\
\hline Biggar & 27 Dec 2011 & -4 & 0 & 20 & 30 & 0 & 10 & overcast & partly cloudy \\
\hline Birch Hills & 20 Dec 2011 & -2 & 2 & 1 & 4 & 3 & 6 & cloudy & cloudy \\
\hline Broadview & 24 Dec 2011 & -2 & 2 & 10 & 10 & 5 & 10 & partly cloudy & mostly clear \\
\hline Cabri & 4 Jan 2012 & 2 & 10 & 0 & 10 & 0 & 2 & clear & clear \\
\hline Candle Lake & 29 Dec 2011 & -7 & -6 & 5 & 10 & 10 & 15 & cloudy & partly cloudy \\
\hline Cater & 1 Jan 2012 & -22 & -10 & 0 & 15 & 10 & 20 & clear & clear \\
\hline Chatsworth S.D. & 3 Jan 2012 & -1 & 1 & 10 & 20 & 1 & 25 & clear & clear \\
\hline Christopher Lake & 22 Dec 2011 & -8 & -8 & 0 & 0 & 6 & 10 & partly cloudy & partly cloudy \\
\hline Churchbridge & 14 Dec 2011 & -12 & -8 & 10 & 15 & 10 & 25 & light snow & overcast \\
\hline Clark's Crossing & 17 Dec 2011 & -8 & -1 & 9 & 13 & 0 & 6 & partly cloudy & cloudy \\
\hline Codette Lake & 17 Dec 2011 & -13 & -4 & 0 & 5 & 0 & 5 & mostly clear & overcast \\
\hline Coronach & 14 Dec 2011 & -6 & -4 & 6 & 44 & 0 & 2 & overcast & overcast \\
\hline Craven & 17 Dec 2011 & -10 & 1 & 0 & 15 & 0 & 5 & clear & clear \\
\hline Creighton & 2 Jan 2012 & -15 & -13 & 9 & 30 & 30 & 60 & light snow & cloudy \\
\hline Crooked Lake & 18 Dec 2011 & -2 & 1 & 10 & 30 & 0 & 10 & light snow & mod.snow \\
\hline Crooked River & 28 Dec 2011 & -8 & -7 & 0 & 0 & 10 & 12 & partly cloudy & partly cloudy \\
\hline Cypress Hills P.P. & 30 Dec 2011 & -2 & 4 & 5 & 20 & 10 & 20 & cloudy & partly cloudy \\
\hline Denholm & 25 Dec 2011 & 0 & 6 & 0 & 20 & 0 & 0 & cloudy & overcast \\
\hline Dorintosh & 3 Jan 2012 & -9 & -2 & 15 & 25 & 4 & 25 & mostly clear & partly cloudy \\
\hline Eastend & 3 Jan 2012 & 4 & 5 & 6 & 30 & 0 & 15 & partly cloudy & mostly clear \\
\hline Endeavour & 18 Dec 2011 & -2 & 0 & 2 & 5 & & & cloudy & partly cloudy \\
\hline Estevan & 30 Dec 2011 & -3 & -1 & 10 & 15 & 0 & 5 & overcast & overcast \\
\hline Estuary North & 3 Jan 2012 & -5 & 5 & 0 & 0 & 0 & 0 & overcast & overcast \\
\hline Fenton & 19 Dec 2011 & -12 & -4 & 2 & 4 & 2 & 5 & mostly clear & mostly clear \\
\hline Fort Qu'Appelle & 16 Dec 2011 & -14 & -4 & 12 & 25 & 0 & 8 & partly cloudy & mostly clear \\
\hline Gardiner Dam & 19 Dec 2011 & -11 & -6 & 10 & 30 & 0 & 3 & partly cloudy & partly cloudy \\
\hline Good Spirit Lake & 29 Dec 2011 & -12 & -10 & 5 & 10 & 0 & 30 & overcast & overcast \\
\hline Grandora & 5 Jan 2012 & -1 & 5 & 6 & 11 & 0 & 1 & clear & clear \\
\hline Grasslands N.P. & 22 Dec 2011 & -12 & -5 & 0 & 5 & 10 & 20 & clear & \\
\hline Grayson & 28 Dec 2011 & -8 & -3 & 0 & 10 & 0 & 10 & mostly clear & overcast \\
\hline Harris & 16 Dec 2011 & -11 & -5 & 15 & 20 & 0 & 10 & overcast & partly cloudy \\
\hline Hazlet & 26 Dec 2011 & 3 & 4 & 40 & 70 & 0 & 5 & partly cloudy & mostly clear \\
\hline Hudson Bay & 26 Dec 2011 & -4 & -2 & 15 & 20 & 30 & 35 & \multicolumn{2}{|c|}{ moderate snow } \\
\hline Indian Head & 30 Dec 2011 & -6 & -2 & 0 & 0 & 15 & 20 & moderate fog & light fog \\
\hline Kenaston & 19 Dec 2011 & -13 & -2 & 8 & 8 & 0 & 0 & clear & overcast \\
\hline Kenosee Lake & 29 Dec 2011 & -5 & 0 & 2 & 22 & 2 & 30 & overcast & overcast \\
\hline Ketchen North & 26 Dec 2011 & -8 & -1 & 5 & 20 & 17 & 23 & cloudy & mod.snow \\
\hline Kilwinnning & 22 Dec 2011 & -9 & -3 & 10 & 25 & 0 & 10 & cloudy & partly cloudy \\
\hline Kindersley North & 26 Dec 2011 & 2 & 3 & 2 & 2 & 0 & 0 & mostly clear & mostly clear \\
\hline Kinloch & 4 Jan 2012 & -7 & 2 & 3 & 6 & 2 & 23 & clear & mostly clear \\
\hline Kyle & 22 Dec 2011 & -14 & -2 & 5 & 15 & 0 & 5 & overcast & overcast \\
\hline
\end{tabular}




\begin{tabular}{|c|c|c|c|c|c|c|c|c|c|}
\hline Locality & Date & 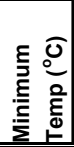 & 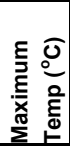 & 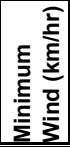 & 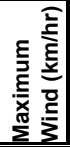 & 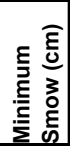 & 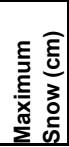 & Sky A.M. & Sky P.M. \\
\hline Lac La Plonge & 28 Dec 2011 & -9 & -16 & 0 & 10 & 20 & 90 & mostly clear & overcast \\
\hline La Ronge & 29 Dec 2011 & -10 & -5 & 2 & 5 & 20 & 20 & cloudy & partly cloudy \\
\hline LMLNWA & 1 Jan 2012 & -15 & -8 & 15 & 30 & 0 & 20 & clear & clear \\
\hline LMLNWA-Nokomis & 17 Dec 2011 & -13 & -2 & 10 & 20 & 0 & 3 & partly cloudy & partly cloudy \\
\hline Love-Torch River & 27 Dec 2011 & -16 & -14 & 0 & 5 & 15 & 20 & overcast & mod.snow \\
\hline Luseland & 2 Jan 2012 & -11 & 4 & 0 & 10 & 0 & 2.5 & cloudy & cloudy \\
\hline Macdowall & 30 Dec 2011 & -11 & -4 & 0 & 0 & 5 & 10 & mostly clear & cloudy \\
\hline MacNutt & 24 Dec 2011 & -3 & 1 & 0 & & 10 & 15 & clear & clear \\
\hline Matador & 3 Jan 2012 & -8 & -2 & 20 & 40 & 0 & 2 & partly cloudy & cloudy \\
\hline Meadow Lake & 26 Dec 2011 & -1 & -3 & 2 & 5 & 0 & 4 & cloudy & cloudy \\
\hline Moose Jaw & 16 Dec 2011 & -11 & -2 & 15 & 25 & 0 & 0 & cloudy & mostly clear \\
\hline Moose Mountain & 3 Jan 2012 & -1 & 3 & 30 & 45 & 1 & 1 & clear & clear \\
\hline Morse & 17 Dec 2011 & -10 & 3 & 5 & 20 & 0 & 20 & clear & mostly clear \\
\hline Nipawin & 30 Dec 2011 & -9 & -8 & 10 & 15 & 10 & 15 & clear & partly cloudy \\
\hline Nisbet Forest NW & 27 Dec 2011 & -6 & 0 & 12 & 20 & & & overcast & heavy snow \\
\hline Nisbet Forest West & 1 Jan 2012 & -16 & -14 & 0 & 15 & 0 & 10 & clear & clear \\
\hline Odessa & 23 Dec 2011 & -8 & 2 & 20 & 29 & 6 & 10 & partly cloudy & clear \\
\hline Outlook & 26 Dec 2011 & -3 & 1 & 20 & 35 & 0 & 0 & overcast & overcast \\
\hline Pierce Lake & 18 Dec 2011 & -6 & -3 & 4 & 16 & 10 & 20 & partly cloudy & partly cloudy \\
\hline Pike Lake & 2 Jan 2012 & -9 & -3 & 22 & 37 & 0 & 10 & partly cloudy & cloudy \\
\hline Prince Albert & 18 Dec 2011 & -2 & 2 & 5 & 5 & 0 & 5 & overcast & clear \\
\hline Prince Albert N.P. & 16 Dec 2011 & -12 & -6 & 10 & 15 & 0 & 15 & cloudy & partly cloudy \\
\hline Qu'Appelle & 9 Jan 2012 & -2 & 6 & 6 & 6 & 4 & 4 & mostly clear & mostly clear \\
\hline Qu'Appelle Dam & 18 Dec 2011 & -4 & 0 & 14 & 27 & 0 & 8 & cloudy & mostly clear \\
\hline Raymore & 25 Dec 2011 & -8 & 4 & 10 & 30 & 0 & 20 & partly cloudy & overcast \\
\hline Regina & 27 Dec 2011 & -4 & 0 & 20 & 30 & 0 & 10 & overcast & cloudy \\
\hline Rokeby & 16 Dec 2011 & -15 & -5 & 10 & 15 & 8 & 15 & light snow & mostly clear \\
\hline Roscommon S.D. & 30 Dec 2011 & -7 & -4 & 2 & 5 & 10 & 15 & light fog & overcast \\
\hline Rouleau & 23 Dec 2011 & -2 & 0 & 2 & 5 & 0 & 1 & mostly clear & mostly clear \\
\hline Round Lake & 17 Dec 2011 & -10 & -1 & 0 & 15 & 0 & 10 & clear & mostly clear \\
\hline Saltcoats & 31 Dec 2011 & -5 & 8 & 17 & 20 & 5 & 10 & mostly clear & mostly clear \\
\hline Sask. Landing P.P. & 30 Dec 2011 & -4 & 4 & 8 & 50 & 0 & 10 & partly cloudy & partly cloudy \\
\hline Sask. River Forks & 17 Dec 2011 & -16 & -5 & 5 & 10 & 5 & 10 & mostly clear & partly cloudy \\
\hline Saskatoon & 26 Dec 2011 & -1 & 4 & 22 & 45 & 0 & 8 & cloudy & partly cloudy \\
\hline Sawyer Lake & 29 Dec 2012 & -13 & -8 & 2 & 5 & 23 & 23 & partly cloudy & partly cloudy \\
\hline Shamrock & 2 Jan 2012 & -5 & -2 & 30 & 45 & 5 & 10 & partly cloudy & cloudy \\
\hline Snowden & 3 Jan 2012 & -4 & 1 & 2 & 2 & 3 & 15 & clear & clear \\
\hline Spinney Hill & 15 Dec 2011 & -11 & -5 & 0 & 10 & 1 & 1 & light fog & light fog \\
\hline Squaw Rapids & 2 Jan 2012 & -30 & -10 & 2 & 12 & 15 & 40 & cloudy & partly cloudy \\
\hline Swift Current & 27 Dec 2011 & -3 & 3 & 45 & 60 & 3 & 8 & partly cloudy & mostly clear \\
\hline Togo & 4 Jan 2012 & -3 & -2 & 5 & 10 & 10 & 20 & mostly clear & mostly clear \\
\hline Weyburn & 17 Dec 2011 & -8 & -1 & 3 & 5 & 0 & 1 & clear & mostly clear \\
\hline White Bear & 26 Dec 2011 & 2 & 3 & 2 & 5 & 0 & 3 & cloudy & cloudy \\
\hline Whitewood & 27 Dec 2011 & -10 & -6 & 15 & 30 & 2 & 20 & overcast & mod.snow \\
\hline Wingard-Ft. Carlton & 29 Dec 2011 & -4 & -4 & 0 & 0 & 5 & 5 & overcast & overcast \\
\hline Yorkton & 17 Dec 2011 & -9 & -6 & 11 & 13 & 10 & 35 & mostly clear & mostly clear \\
\hline
\end{tabular}


Table 2-1. Effort and Habitat Coverage. Wild Fruit: $p=$ poor, $f=$ fair, $g=g o o d, e=e x c e l l e n t$

\begin{tabular}{|c|c|c|c|c|c|c|c|c|c|c|c|c|c|c|c|c|c|c|c|c|}
\hline Locality & 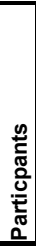 & 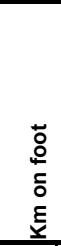 & 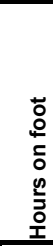 & 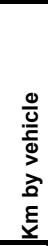 & 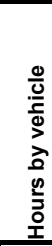 & 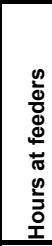 & 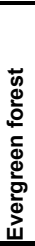 & 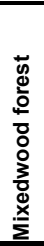 & 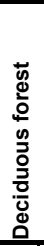 & 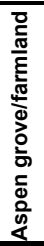 & 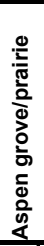 & 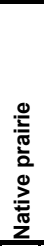 & 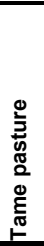 & 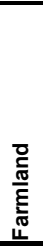 & 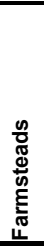 & $\begin{array}{l}\text { 졿 } \\
\text { 은 }\end{array}$ & 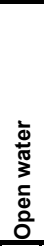 & $\begin{array}{l}\frac{c}{\sqrt{\pi}} \\
\frac{1}{\pi} \\
\frac{0}{\bar{N}} \\
\underline{\underline{\alpha}}\end{array}$ & 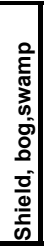 & 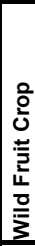 \\
\hline Archerwill & 6 & 10.0 & 4.5 & 100 & 5.0 & 6.0 & & 25 & & & & & & 50 & \begin{tabular}{|l|}
25 \\
\end{tabular} & & & & & \\
\hline Avonlea & 2 & 5.0 & 3.0 & 114 & 4.5 & 0.5 & & & 15 & & & 5 & 5 & 35 & 15 & 25 & & & & $p$ \\
\hline Balgonie & 12 & 2.0 & 0.5 & 414 & 19.0 & & & & & 35 & 5 & & & 50 & 5 & 5 & & & & $g$ \\
\hline Bangor & 8 & 3.0 & 2.0 & 30 & 3.0 & 32.0 & & & & 10 & & & & & 90 & & & & & $\mathrm{p}$ \\
\hline Biggar & 9 & 18.0 & 9.0 & 447 & 12.3 & 5.0 & & & & 19 & & & & 49 & & 32 & & & & g \\
\hline Birch Hills & 5 & 9.0 & 1.5 & 114 & 22.0 & 6.0 & & & 5 & 40 & & & 2 & 44 & 3 & 5 & 1 & & & $f$ \\
\hline Broadview & 3 & 2.5 & 1.5 & 115 & 5.0 & 2.0 & & & 20 & 35 & & 5 & & 25 & 5 & 10 & & & & $p$ \\
\hline Cabri & 3 & 3.5 & 2.0 & 124 & 5.0 & & & & & & & & & & & & & & & $p$ \\
\hline Candle Lake & 4 & 5.0 & 5.0 & 178 & 8.0 & & 20 & 20 & 30 & & & & & & & 30 & & & & $\mathrm{p}$ \\
\hline Cater & 4 & & & 234 & 8.0 & 1.0 & 5 & 10 & 5 & 15 & & & 5 & 50 & 5 & 5 & & & & $g$ \\
\hline Chatsworth S.D. & 6 & 2.0 & 3.0 & 42 & 6.0 & & & & & \begin{tabular}{|l|}
80 \\
\end{tabular} & & 10 & & & \begin{tabular}{|l|}
10 \\
\end{tabular} & & & & & $f$ \\
\hline Christopher Lake & 1 & 10.0 & 2.5 & & & & & 65 & & 35 & & & & & & & & & & $p$ \\
\hline Churchbridge & 13 & & & 128 & 12.0 & 66.0 & & & & \begin{tabular}{|l|}
10 \\
\end{tabular} & & 15 & & \begin{tabular}{|l|}
72 \\
\end{tabular} & 2 & 1 & & & & $f$ \\
\hline Clark's Crossing & 24 & 17.0 & 17.4 & 591 & 27.6 & 2.0 & & & & 22 & 3 & 1 & & 28 & 16 & 24 & 1 & 5 & & $p$ \\
\hline Codette Lake & 2 & 2.0 & 1.0 & 100 & 4.0 & & 5 & 10 & 20 & 20 & & & & 15 & 25 & & 5 & & & $g$ \\
\hline Coronach & 3 & 0.5 & 1.5 & 130 & 5.3 & & & & & 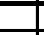 & & 5 & 5 & 45 & 5 & 10 & 30 & & & \\
\hline Craven & 35 & 31.0 & 16.0 & 427 & 21.0 & 7.0 & & & & 15 & & 5 & 10 & 45 & 10 & 15 & & & & \\
\hline Creighton & 2 & 1.0 & \begin{tabular}{|l}
1.0 \\
\end{tabular} & 142 & 6.0 & 2.0 & 10 & \begin{tabular}{|l|}
10 \\
\end{tabular} & \begin{tabular}{|l|}
10 \\
\end{tabular} & & & & & & & \begin{tabular}{|l|}
20 \\
\end{tabular} & & & 50 & $\mathrm{e}$ \\
\hline Crooked Lake & 1 & 0.5 & 0.5 & 126 & 3.5 & & & & & 40 & & & & & & 10 & 50 & & & $\mathrm{f}$ \\
\hline Crooked River & 5 & 0.5 & 5.0 & 25 & 0.5 & 5.0 & & 40 & & & & & & 40 & 20 & & & & & $\mathrm{~g}$ \\
\hline Cypress Hills P.P. & 6 & 8.0 & 4.5 & 15 & 1.0 & & 40 & 40 & 8 & & & 10 & & 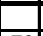 & 2 & & & & & $g$ \\
\hline Denholm & 3 & & & 304 & 6.5 & 3.0 & & & & 15 & & & 5 & 70 & 5 & 5 & & & & $g$ \\
\hline Dorintosh & 2 & 10.0 & 3.0 & 100 & 3.0 & 2.0 & 60 & & & & & & & 40 & & & & & & $p$ \\
\hline Eastend & 8 & 6.0 & 9.0 & 106 & 15.0 & 5.0 & & 5 & & 20 & 50 & 10 & 5 & 5 & & 5 & $\mathrm{t}$ & & & $p$ \\
\hline Endeavour & 1 & & & 45 & 3.0 & 4.0 & & 10 & & 30 & & & & & 50 & 10 & & & & $g$ \\
\hline Estevan & 5 & 5.0 & 4.0 & 177 & 5.8 & 3.0 & & & & & & & & 10 & 9 & 36 & 15 & 30 & & $g$ \\
\hline Estuary North & 2 & 4.0 & 4.0 & 50 & 4.0 & & & & 50 & & & 25 & 10 & 10 & 5 & & & & & $f$ \\
\hline Fenton & 3 & 2.0 & 1.0 & 122 & 5.0 & & & & & & & & & & & & & & & $p$ \\
\hline Fort Qu'Appelle & 22 & & & & & & & & 2 & 15 & & & & 40 & 1 & 41 & 1 & & & $p$ \\
\hline Gardiner Dam & 8 & 33.0 & 16.3 & 428 & 15.0 & 1.0 & & & 5 & 3 & & & & 37 & 15 & 3 & 32 & 5 & & \\
\hline Good Spirit Lake & 5 & & & 106 & 4.0 & 3.0 & & 5 & 5 & 5 & & 5 & 5 & 30 & 5 & 40 & & & & $f$ \\
\hline Grandora & 3 & 2.0 & 2.0 & 23 & 5.0 & 1.5 & & & & 90 & & & & & 10 & & & & & \\
\hline Grasslands N.P. & 16 & 5.0 & 2.0 & 210 & 20.0 & & & & & & & 70 & 5 & 15 & 2 & 3 & & 5 & & \\
\hline Grayson & 6 & 10.0 & 2.0 & 104 & 5.0 & 4.0 & & & & 20 & & & & 20 & 20 & 20 & 20 & & & \\
\hline Harris & 2 & 5.0 & 2.8 & 190 & 6.3 & & & & & 17 & & & & 53 & & 30 & & & & $f$ \\
\hline Hazlet & 2 & 1.0 & 0.8 & 185 & 5.1 & & & & & & & \begin{tabular}{|l|}
10 \\
\end{tabular} & & \begin{tabular}{|l|}
80 \\
\end{tabular} & 5 & 5 & & & & $f$ \\
\hline Hudson Bay & 12 & & & 12 & 1.0 & 15.0 & & 12 & & & & & & & 76 & 12 & & & & $g$ \\
\hline Indian Head & 21 & 2.0 & 1.0 & 155 & 5.0 & 15.0 & & & & & & & & 20 & 30 & 40 & 10 & & & $p$ \\
\hline Kenaston & 4 & & & 83 & 6.0 & & & & & 10 & 10 & & & 70 & 10 & & & & & \\
\hline Kenosee Lake & 2 & & & 124 & 4.5 & 0.5 & & 5 & 45 & 20 & & & & & & 30 & & & & $p$ \\
\hline Ketchen North & 1 & 1.0 & 1.5 & 50 & 2.0 & 6.0 & & & & & 30 & & 20 & 20 & 20 & 10 & & & & \\
\hline Kilwinnning & 2 & 1.0 & 0.5 & 151 & 6.8 & & 5 & 15 & 5 & 50 & & & 5 & 20 & & & & & & $p$ \\
\hline Kindersley North & 2 & & & & 5.0 & & & & & & & & & & & & & & & \\
\hline Kinloch & 5 & 6.0 & 3.0 & 74 & 4.0 & 12.0 & & 60 & 20 & & & & & & 20 & & & & & $g$ \\
\hline Kyle & 4 & 7.0 & 4.0 & 260 & 12.0 & 2.0 & & & & 2 & & 25 & 5 & 63 & 1 & 2 & & 2 & & $p$ \\
\hline
\end{tabular}


Table 2-2. Effort and Habitat Coverage. Wild Fruit: $p=p o o r, f=f a i r, g=g o o d, e=e x c e l l e n t$.

\begin{tabular}{|c|c|c|c|c|c|c|c|c|c|c|c|c|c|c|c|c|c|c|c|c|}
\hline Locality & 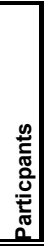 & 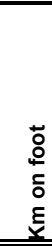 & 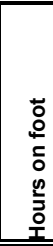 & 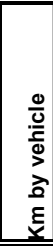 & 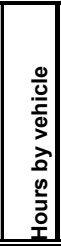 & 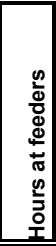 & 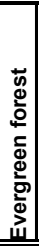 & 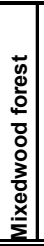 & 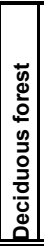 & 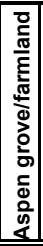 & 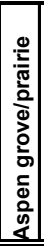 & 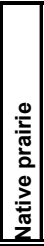 & 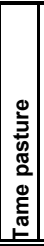 & 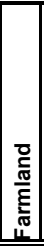 & 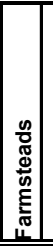 & $\begin{array}{l}\frac{c}{\mathbb{1}} \\
\text { 온 } \\
\end{array}$ & \begin{tabular}{l}
$\frac{1}{ \pm}$ \\
$\overline{0}$ \\
3 \\
\multicolumn{1}{c}{} \\
$\bar{\Phi}$ \\
0 \\
\end{tabular} & \begin{tabular}{|c|}
$\frac{c}{\pi}$ \\
$\frac{\pi}{\frac{\pi}{J}}$ \\
$\frac{.}{\bar{\alpha}}$ \\
\end{tabular} & 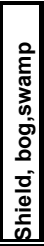 & 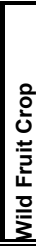 \\
\hline Lac La Plonge & 4 & $\overline{1} 1$ & 0.5 & 47.6 & 5.75 & 1.5 & & 40 & 40 & & & & & & & 20 & & & & p \\
\hline \begin{tabular}{|l} 
La Ronge \\
\end{tabular} & 1 & & & 36 & 2.0 & 2.0 & 30 & \begin{tabular}{|l|}
10 \\
\end{tabular} & & & & & & & & \begin{tabular}{|l|}
60 \\
\end{tabular} & & & & $g$ \\
\hline LMLNWA & 2 & 1.0 & 0.5 & 153 & 7.0 & 0.8 & & & & 10 & & 15 & & 50 & 5 & 20 & & & & $f$ \\
\hline LMLNWA-Nokomis & 1 & 4.0 & 1.3 & 135 & 4.8 & 0.8 & & & & 10 & 5 & 20 & 10 & 40 & 1 & 1 & & 13 & & $p$ \\
\hline Love-Torch River & 26 & 2.0 & 4.0 & 390 & 10.8 & 28.0 & 10 & \begin{tabular}{|l|}
10 \\
\end{tabular} & & 60 & & & & & 10 & \begin{tabular}{|l|}
10 \\
\end{tabular} & & & & $p$ \\
\hline Luseland & 7 & 7.0 & 4.0 & 237 & 6.0 & 4.0 & & & & 35 & 10 & 5 & 5 & 30 & 5 & 10 & & & & $f$ \\
\hline Macdowall & 7 & 0.5 & 0.3 & 110 & 7.3 & 10.0 & 5 & \begin{tabular}{|l|}
25 \\
\end{tabular} & \begin{tabular}{|l|}
10 \\
\end{tabular} & 5 & & & & 25 & 30 & & & & & $p$ \\
\hline MacNutt & 2 & & & 150 & 5.5 & 4.0 & & & 20 & 30 & 10 & 2 & 20 & & 8 & 10 & & & & g \\
\hline Matador & 2 & 2.0 & 1.0 & 115 & 5.3 & & & & & & & 85 & 5 & 5 & & & & 5 & & $f$ \\
\hline Meadow Lake & 6 & 5.0 & 4.0 & 121 & 4.0 & 8.0 & & \begin{tabular}{|l|}
50 \\
\end{tabular} & & & & & & 30 & & 20 & & & & $p$ \\
\hline Moose Jaw & 3 & 7.5 & 3.8 & 160 & 6.5 & 1.0 & & & & & & $\mathrm{t}$ & & 15 & $\mathrm{t}$ & 60 & \begin{tabular}{|l|}
25 \\
\end{tabular} & & & $f$ \\
\hline Moose Mountain & 4 & & & 199 & 8.0 & & 5 & & 5 & 20 & 5 & 10 & \begin{tabular}{|l|}
10 \\
\end{tabular} & 25 & 5 & 5 & \begin{tabular}{|l|}
10 \\
\end{tabular} & & & $f$ \\
\hline Morse & 11 & 6.0 & 4.5 & 543 & 28.3 & 1.0 & & & & & & & 3 & 90 & 7 & & & & & $p$ \\
\hline Nipawin & 14 & 2.5 & 2.5 & 204 & 10.0 & 4.0 & 20 & 10 & & 5 & & & & 20 & 10 & 30 & 5 & & & $\mathrm{~g}$ \\
\hline Nisbet Forest NW & 3 & 2.0 & 1.0 & & & 4.5 & & & & & & & & 20 & 80 & & & & & g \\
\hline Nisbet Forest West & 4 & 1.0 & 0.5 & 120 & 4.5 & & 5 & & 30 & & & & 10 & 30 & 10 & 15 & & & & $\mathrm{~g}$ \\
\hline Odessa & 3 & & & 30 & 20.0 & & & & & 25 & 20 & 10 & 10 & 25 & 10 & & & & & $p$ \\
\hline Outlook & 2 & 3.0 & 2.0 & 128 & 4.8 & 0.5 & & & & & 5 & & 5 & 35 & 5 & 45 & 5 & & & $f$ \\
\hline Pierce Lake & 4 & & & 150 & 4.5 & & 10 & 30 & 30 & 10 & & & & 10 & & & 10 & & & $p$ \\
\hline Pike Lake & 46 & 32.0 & 25.0 & 476 & 23.5 & 13.0 & & & 15 & 10 & & & 5 & 15 & 15 & 40 & & & & $p$ \\
\hline Prince Albert & 25 & 12.2 & 7.3 & 418 & 23.0 & 2.0 & & & & & & & & & & & & & & $p$ \\
\hline Prince Albert N.P. & 24 & 168 & 30.5 & 35 & 1.8 & & & 95 & & & & & & & & 5 & & & & \\
\hline Qu'Appelle & 2 & & & 40 & 0.3 & 0.7 & & & & & & & & 35 & 65 & & & & & $g$ \\
\hline Qu'Appelle Dam & 10 & 4.0 & 4.8 & 545 & 21.9 & & & 2 & 13 & 10 & 5 & 5 & 10 & 15 & 10 & 20 & 10 & & & $p$ \\
\hline Raymore & 2 & 0.5 & 1.0 & 136 & 8.0 & 0.5 & & & & 35 & & & & 35 & 10 & 20 & & & & $f$ \\
\hline Regina & 34 & 53.0 & 23.0 & 493 & 35.0 & 10.0 & & & & 5 & & & & 25 & 5 & 65 & & & & $g$ \\
\hline Rokeby & 6 & & & 50 & 8.0 & 3.0 & & & & 4 & & & & 95 & 1 & & & & & \\
\hline Roscommon S.D. & 8 & 0.5 & 1.0 & 90 & 4.0 & 18.0 & & & & 30 & & & & 60 & 10 & & & & & $g$ \\
\hline Rouleau & 4 & 8.0 & 1.0 & 213 & 5.5 & 0.5 & & & & & & & & 80 & \begin{tabular}{|l|}
10 \\
\end{tabular} & 10 & & & & $p$ \\
\hline Round Lake & 3 & 1.0 & 0.5 & 160 & 6.0 & 4.0 & & & 25 & 45 & & & & & & & 30 & & & $f$ \\
\hline Saltcoats & 16 & 1.5 & 1.5 & 198 & 6.5 & & & & & & & & & 40 & 50 & 10 & & & & $f$ \\
\hline Sask. Landing P.P. & 5 & 6.0 & 2.0 & 250 & 8.0 & & & & & 20 & & 25 & 5 & 39 & 1 & & & 10 & & $p$ \\
\hline Sask. River Forks & 4 & 6.0 & 2.5 & 84 & 6.0 & & 15 & 20 & 20 & 15 & & & 5 & 15 & 5 & & 5 & & & $p$ \\
\hline Saskatoon & 96 & 100 & 46.6 & 806 & 55.6 & 110 & & & & 10 & 1 & 1 & 4 & 11 & 9 & 57 & 3 & 4 & & $p$ \\
\hline Sawyer Lake & 4 & 14.0 & 6.0 & 28 & 10.0 & 4 & & 30 & & 20 & & & 5 & 40 & 5 & & & & & $f$ \\
\hline Shamrock & 7 & 4.0 & 4.0 & 312 & 10.5 & & & & & & & & 5 & 75 & 20 & & & & & \\
\hline Snowden & 14 & & 3.5 & 100 & 2.5 & 2.5 & & \begin{tabular}{|l|}
10 \\
\end{tabular} & & & & & & 30 & \begin{tabular}{|l|}
50 \\
\end{tabular} & \begin{tabular}{|l|}
10 \\
\end{tabular} & & & & $g$ \\
\hline Spinney Hill & 2 & 1.0 & 0.5 & 152 & 6.5 & & & & 35 & 25 & & 5 & & 35 & & & & & & $p$ \\
\hline Squaw Rapids & 2 & 1.0 & 0.8 & 93 & 5.8 & & & 93 & & & & & & 2 & & & 5 & & & $p$ \\
\hline Swift Current & 21 & 14.0 & 9.5 & 427 & 29.0 & 9.5 & & & & & & & & 20 & 10 & 70 & & & & \\
\hline Togo & 10 & 4.0 & 2.0 & 60 & 2.5 & 5.5 & & 10 & 20 & 30 & 10 & 5 & & 25 & & & & & & f \\
\hline Weyburn & 14 & 2.0 & 3.0 & 507 & 14.0 & & & & & & 5 & 15 & \begin{tabular}{|l|}
10 \\
\end{tabular} & 60 & 5 & 5 & & & & $p$ \\
\hline White Bear & 2 & & & 110 & 3.0 & & & & & & & 20 & 5 & 73 & 1 & & & 1 & & $p$ \\
\hline Whitewood & 18 & 2.5 & 1.5 & 541 & 15.5 & 37.5 & & & & 50 & & & & & 10 & 40 & & & & $p$ \\
\hline Wingard-Ft. Carlton & 6 & 1.0 & 0.3 & 80 & 5.3 & 10.0 & & 5 & 15 & 10 & & & & 25 & 45 & & & & & $f$ \\
\hline Yorkton & 26 & 1.0 & 1.0 & 116 & 10.0 & 16.0 & & & & 30 & & & & & & 70 & & & & $\mathrm{e}$ \\
\hline
\end{tabular}


Table 3-1. Species found on 8 or more counts; ()$=$ seen during Count Period (CP).

\begin{tabular}{|c|c|c|c|c|c|c|c|c|c|c|c|}
\hline Map Number & 1 & 2 & 3 & 4 & 5 & 6 & 7 & 8 & 9 & 10 & 11 \\
\hline Species & 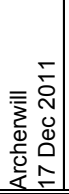 & 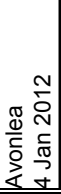 & 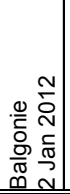 & 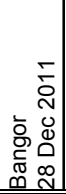 & 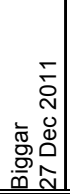 & 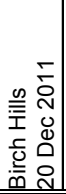 & 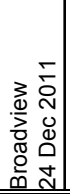 & 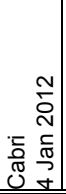 & 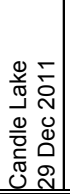 & 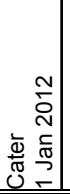 & 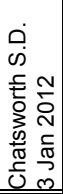 \\
\hline \multicolumn{12}{|l|}{ Canada Goose } \\
\hline Mallard & & & & & 8 & & & & & & \\
\hline \multicolumn{12}{|l|}{ Common Goldeneye } \\
\hline \multicolumn{12}{|l|}{ Common Merganser } \\
\hline Gray Partridge & & & & 1 & & (3) & & & & & 11 \\
\hline \multicolumn{12}{|l|}{ Ring-necked Pheasant } \\
\hline Ruffed Grouse & 2 & & & & & (1) & 2 & & & (2) & 2 \\
\hline Sharp-tailed Grouse & & 1 & 5 & 21 & 26 & & & 1 & & & 8 \\
\hline Bald Eagle & (1) & & & & & & & & & & 1 \\
\hline \multicolumn{12}{|l|}{ Northern Goshawk } \\
\hline Rough-legged Hawk & & & & & 5 & & & & & & \\
\hline Golden Eagle & & 1 & & & 2 & & & & & & \\
\hline \multicolumn{12}{|l|}{ Merlin } \\
\hline \multicolumn{12}{|l|}{ Gyrfalcon } \\
\hline Rock Pigeon & 21 & 135 & 276 & 7 & 271 & 121 & 59 & 39 & & 2 & 12 \\
\hline Eurasian Collared-Dove & & & & & 21 & & & 30 & & & \\
\hline Mourning Dove & & & & & & & (1) & & & & \\
\hline Great Horned Owl & & (1) & & 2 & 4 & 2 & 1 & 1 & & & \\
\hline Snowy Owl & & & 5 & & 2 & & 1 & 11 & & & \\
\hline Downy Woodpecker & & 1 & 3 & 9 & 1 & 3 & (1) & & 1 & & 4 \\
\hline Hairy Woodpecker & 4 & 1 & & 11 & 1 & 9 & 3 & & 2 & 6 & 3 \\
\hline Northern Flicker & & & & & 1 & & & & & & \\
\hline Pileated Woodpecker & (1) & & & & & & & & 3 & & (1) \\
\hline Northern Shrike & & & & & & 2 & & & . & & \\
\hline Gray Jay & (2) & & & & & & & & 1 & & \\
\hline Blue Jay & 10 & & & & 1 & 4 & 3 & & 5 & 4 & \\
\hline Black-billed Magpie & 46 & 14 & 82 & 41 & 124 & 88 & 19 & 42 & 4 & 30 & 20 \\
\hline Common Raven & 67 & $(1)$ & 30 & 31 & 88 & 16 & 16 & 1 & 224 & 159 & 14 \\
\hline \multicolumn{12}{|l|}{ Horned Lark } \\
\hline Black-capped Chickadee & 29 & & 68 & 61 & 29 & 37 & 17 & 2 & 25 & 21 & 35 \\
\hline Boreal Chickadee & 2 & & & & & & & & 7 & & \\
\hline Red-breasted Nuthatch & 1 & 2 & & & 4 & & & & 11 & & \\
\hline White-breasted Nuthatch & 5 & 2 & 2 & 3 & & & 2 & & 1 & 2 & 4 \\
\hline \multirow{2}{*}{\multicolumn{12}{|c|}{\begin{tabular}{|l|} 
Brown Creeper \\
Golden-crowned Kinglet \\
\end{tabular}}} \\
\hline & & & & & & & 2 & & & & \\
\hline American Robin & & & & & 2 & & & & & & \\
\hline European Starling & & 21 & 21 & & & & (4) & 9 & & & 2 \\
\hline Bohemian Waxwing & & & 16 & & 291 & 190 & 50 & & & 2 & \\
\hline Snow Bunting & 330 & & 89 & 40 & 67 & (31) & 1 & 2 & & 566 & 50 \\
\hline Dark-eyed Junco & & 2 & & & 3 & & & & & & \\
\hline Pine Grosbeak & 42 & & & 7 & 6 & 31 & & & 29 & 81 & \\
\hline House Finch & & (3) & 2 & & 65 & & & & & & \\
\hline White-winged Crossbill & & & & & & & & & 8 & & \\
\hline Common Redpoll & 124 & 73 & 538 & 57 & 615 & 221 & 192 & 35 & 107 & 188 & 85 \\
\hline Hoary Redpoll & & & 1 & & 2 & 2 & & & 1 & & \\
\hline \multicolumn{12}{|l|}{ Pine Siskin } \\
\hline American Goldfinch & & (1) & & & 1 & (1) & & & & & \\
\hline Evening Grosbeak & 67 & & & & & & & & 2 & & \\
\hline House Sparrow & & 70 & 376 & 126 & 655 & 59 & 108 & 631 & & \begin{tabular}{l|l}
59 \\
\end{tabular} & 38 \\
\hline Total Birds Count Day & 750 & 323 & 1517 & 417 & 2298 & 785 & 480 & 805 & 434 & 1120 & 289 \\
\hline Total Birds only in CP & 5 & 8 & 0 & 0 & 0 & 37 & 6 & 0 & 0 & 2 & 1 \\
\hline Total Species Count Day & 14 & 12 & 16 & 14 & 28 & 14 & 16 & 13 & 18 & 12 & 15 \\
\hline Total Species only in CP & 4 & 6 & 0 & 0 & 0 & 5 & 3 & 0 & 0 & 1 & 1 \\
\hline
\end{tabular}


Table 3-2. Species found on 8 or more counts; ( ) = seen during Count Period (CP).

\begin{tabular}{|c|c|c|c|c|c|c|c|c|c|c|c|}
\hline Map Number & 12 & 13 & 14 & 15 & 16 & 17 & \begin{tabular}{|l|l|}
18 \\
\end{tabular} & 19 & 20 & 21 & 22 \\
\hline Species & 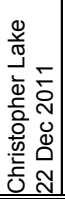 & 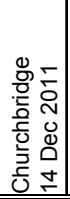 & 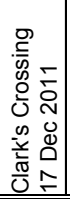 & 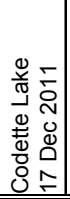 & 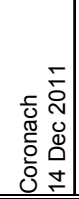 & 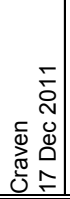 & 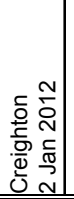 & 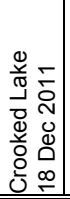 & 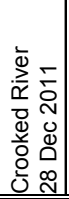 & 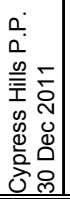 & 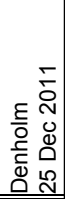 \\
\hline Canada Goose & & & 750 & & 9187 & & & \begin{tabular}{ll|}
464 \\
\end{tabular} & & & \\
\hline Mallard & & & 2 & & 5325 & 1 & & 47 & & & \\
\hline Common Goldeneye & & & 2 & & 4 & 15 & & & & & \\
\hline Common Merganser & & & & & & & & & & & \\
\hline Gray Partridge & & & 23 & 1 & & 10 & & & & & (14) \\
\hline Ring-necked Pheasant & & & & & & 1 & & & & & \\
\hline Ruffed Grouse & & 1 & & 2 & & 1 & & & 1 & & \\
\hline Sharp-tailed Grouse & & 14 & 38 & & 56 & 34 & 1 & & & & 9 \\
\hline Bald Eagle & & & 4 & 1 & & & & 2 & & & 1 \\
\hline Northern Goshawk & & & & & & & 2 & & & & \\
\hline Rough-legged Hawk & & & & & & & & 1 & & 1 & \\
\hline Golden Eagle & & & & & 1 & 1 & & 1 & & 1 & \\
\hline Merlin & & & & & & & & & & & 1 \\
\hline Gyrfalcon & & & & & & & & & & & \\
\hline Rock Pigeon & & 6 & 341 & & 5 & 79 & 142 & 23 & & & 91 \\
\hline Eurasian Collared-Dove & & & & & 50 & & & & & & \\
\hline Mourning Dove & & & & & & & & & & & \\
\hline Great Horned Owl & & & 5 & & & 1 & & & & & (1) \\
\hline Snowy Owl & & & 3 & & 7 & 13 & & & & & \\
\hline Downy Woodpecker & 1 & 11 & 6 & 2 & & 21 & (1) & & 3 & & 2 \\
\hline Hairy Woodpecker & 2 & 10 & 10 & 3 & & 11 & 1 & & 3 & 1 & 2 \\
\hline Northern Flicker & & & 3 & & & & & & & 1 & \\
\hline Pileated Woodpecker & & & & & & & & & & & \\
\hline Northern Shrike & & & & & & & & 1 & & & \\
\hline Gray Jay & & & & & & & 2 & & 2 & & \\
\hline Blue Jay & & 2 & 10 & 6 & & 42 & & 1 & 14 & & 5 \\
\hline Black-billed Magpie & 6 & 25 & 401 & 30 & 1 & 138 & 6 & 7 & 8 & 5 & 66 \\
\hline Common Raven & & 53 & 218 & 30 & & 95 & 443 & 11 & 60 & 3 & 39 \\
\hline Horned Lark & 8 & & & & 17 & & & & & & \\
\hline Black-capped Chickadee & 13 & 57 & 148 & 31 & & 202 & 5 & 5 & 33 & 26 & 12 \\
\hline Boreal Chickadee & & & & & & & 7 & & & & \\
\hline Red-breasted Nuthatch & & & 3 & & & 4 & & & 2 & 10 & \\
\hline White-breasted Nuthatch & 2 & 4 & & 1 & & 15 & & 2 & 4 & & \\
\hline Brown Creeper & & & & & & 2 & & & & & \\
\hline Golden-crowned Kinglet & & & & & & & & & & & \\
\hline American Robin & & & 1 & & & (1) & & & & & \\
\hline European Starling & & & 115 & & 7 & 210 & & & 4 & & \\
\hline Bohemian Waxwing & & 21 & & 15 & & 72 & & & & 60 & $(12)$ \\
\hline Snow Bunting & & 130 & & & & (2) & & 10 & & & $(75)$ \\
\hline Dark-eyed Junco & & & & & & 2 & & & & & \\
\hline Pine Grosbeak & 7 & 8 & 30 & 42 & & (2) & 31 & & 29 & & 6 \\
\hline House Finch & & & 20 & & & 12 & & & & & \\
\hline White-winged Crossbill & & & & & & & (1) & & & & \\
\hline Common Redpoll & 40 & 65 & 406 & 145 & 12 & 273 & 62 & 576 & & 33 & 242 \\
\hline Hoary Redpoll & & 10 & 5 & 1 & & & 6 & & & & 2 \\
\hline Pine Siskin & & & & & & & & & & & \\
\hline American Goldfinch & & & & & & (10) & & & & & \\
\hline Evening Grosbeak & 43 & & & 19 & & & 25 & & 66 & & \\
\hline House Sparrow & & 104 & 1221 & 43 & 54 & 305 & 2 & & 18 & 3 & 61 \\
\hline Total Birds Count Day & 122 & 523 & 3771 & 372 & 14734 & 1562 & 755 & 1156 & 247 & 149 & 539 \\
\hline Total Birds only in CP & 0 & 0 & 0 & 0 & 0 & 15 & 2 & 0 & 0 & 0 & 102 \\
\hline Total Species Count Day & 9 & 17 & 29 & 16 & 18 & 27 & 15 & 17 & 14 & 14 & 14 \\
\hline Total Species only in CP & 0 & 0 & 0 & 0 & 0 & 4 & 2 & 0 & 0 & 0 & 4 \\
\hline
\end{tabular}


Table 3-3. Species found on 8 or more counts; ( ) = seen during Count Period (CP).

\begin{tabular}{|c|c|c|c|c|c|c|c|c|c|c|c|}
\hline \begin{tabular}{|l} 
Map Number \\
\end{tabular} & 23 & 24 & 25 & 26 & 27 & 28 & 29 & 30 & 31 & 32 & 33 \\
\hline Species & 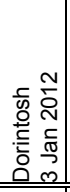 & 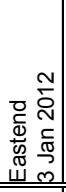 & 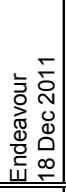 & 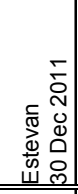 & 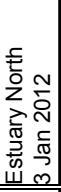 & 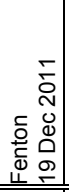 & 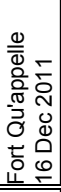 & 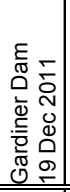 & 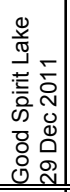 & 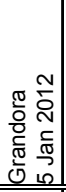 & 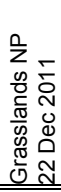 \\
\hline Canada Goose & & & & 11117 & & & 420 & 872 & & & \\
\hline Mallard & (1) & 6 & & 2581 & & & 18 & 1831 & & & \\
\hline Common Goldeneye & & & & 39 & & & 20 & 191 & & & \\
\hline Common Merganser & & & & 2 & & & 8 & 77 & & & \\
\hline Gray Partridge & & 9 & & & & & & 19 & & & 22 \\
\hline Ring-necked Pheasant & & 13 & & 114 & 10 & & & & & & 45 \\
\hline Ruffed Grouse & (3) & & (1) & & & & & & 2 & & \\
\hline Sharp-tailed Grouse & (2) & 8 & & 4 & & & 8 & 32 & 11 & & 187 \\
\hline Bald Eagle & & 1 & & 1 & & & 1 & 45 & & $(2)$ & \\
\hline Northern Goshawk & & & & 1 & 2 & & (1) & & & & \\
\hline Rough-legged Hawk & & & & 1 & 1 & & & & & & \\
\hline Golden Eagle & & 1 & & 4 & & & & 2 & & & 1 \\
\hline Merlin & & & & & & & & & & & 1 \\
\hline Gyrfalcon & & & & (1) & & & & & & & \\
\hline Rock Pigeon & & 10 & 20 & 24 & & 38 & 123 & 124 & & & 65 \\
\hline Eurasian Collared-Dove & & 38 & & 33 & & & 2 & 6 & & & \\
\hline Mourning Dove & & & & 1 & & & & & & & \\
\hline Great Horned Owl & & 4 & & & & & (1) & 5 & & & 1 \\
\hline Snowy Owl & (1) & 3 & & 4 & 1 & & & 3 & & & 4 \\
\hline Downy Woodpecker & 2 & 1 & 1 & 2 & 1 & 1 & 11 & & 2 & & 1 \\
\hline Hairy Woodpecker & 2 & 1 & 2 & 2 & 1 & 2 & 6 & & 2 & 1 & \\
\hline Northern Flicker & & 1 & & (1) & 2 & & & & & & \\
\hline Pileated Woodpecker & (1) & & & & & & & & 2 & & \\
\hline Northern Shrike & & 1 & & & & 2 & 1 & & & & 2 \\
\hline Gray Jay & 4 & & 1 & & & 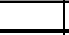 & & & & & \\
\hline Blue Jay & 5 & 1 & 4 & (3) & 5 & 2 & 25 & 7 & 11 & & \\
\hline Black-billed Magpie & 2 & 78 & 8 & 7 & 18 & 55 & 101 & 132 & 24 & & 51 \\
\hline Common Raven & 12 & 5 & 15 & 5 & 1 & 21 & 115 & 39 & 21 & 12 & \\
\hline Horned Lark & & & & & & & & & & & 28 \\
\hline Black-capped Chickadee & 15 & 37 & 26 & 10 & 12 & 31 & 128 & 14 & 53 & 4 & \\
\hline \multicolumn{12}{|l|}{ Boreal Chickadee } \\
\hline Red-breasted Nuthatch & 2 & 19 & & & & & 1 & 2 & 3 & & \\
\hline White-breasted Nuthatch & 2 & & 2 & 1 & & 1 & 13 & & 3 & 1 & \\
\hline Brown Creeper & & & & & & & & 1 & & 1 & \\
\hline \multicolumn{12}{|l|}{ Golden-crowned Kinglet } \\
\hline American Robin & & & & & & & & & & (1) & \\
\hline European Starling & & 25 & 6 & 62 & & & 24 & 4 & & & \\
\hline \begin{tabular}{|l|} 
Bohemian Waxwing \\
\end{tabular} & & & & (12) & 15 & 177 & 44 & 62 & & & \\
\hline Snow Bunting & $(100)$ & & 35 & 12 & & & & 16 & 120 & & 121 \\
\hline Dark-eyed Junco & & 7 & & & & & 3 & 2 & & & \\
\hline Pine Grosbeak & 3 & 8 & 25 & & 27 & 39 & & 24 & 31 & & \\
\hline \begin{tabular}{|l} 
House Finch \\
\end{tabular} & & 2 & & $(2)$ & & & 12 & & & & \\
\hline White-winged Crossbill & & 17 & & & & & & & & & \\
\hline Common Redpoll & 25 & 61 & 65 & 385 & 22 & 138 & 761 & 91 & 12 & & 1 \\
\hline Hoary Redpoll & & & 1 & & & & & 1 & & & \\
\hline \multicolumn{12}{|l|}{ Pine Siskin } \\
\hline American Goldfinch & & & & (3) & & & 3 & & & & \\
\hline Evening Grosbeak & 5 & & 47 & & & & & & & & \\
\hline House Sparrow & & 151 & 50 & 145 & 40 & 113 & 104 & 490 & 2 & 14 & 49 \\
\hline Total Birds Count Day & 79 & 510 & 308 & 14599 & 158 & 620 & 1973 & 4210 & 300 & 33 & 582 \\
\hline Total Birds only in CP & 108 & 0 & 1 & 23 & 0 & 0 & 4 & 0 & 0 & 3 & 0 \\
\hline Total Species Count Day & 12 & 28 & 16 & 35 & 15 & 13 & 29 & 37 & 16 & 6 & 17 \\
\hline Total Species only in CP & 6 & 0 & 1 & 7 & 0 & 0 & 4 & 0 & 0 & 2 & 0 \\
\hline
\end{tabular}


Table 3-4. Species found on 8 or more counts; ( ) = seen during Count Period (CP).

\begin{tabular}{|c|c|c|c|c|c|c|c|c|c|c|c|}
\hline Map Number & 34 & 35 & 36 & 37 & 38 & 39 & 40 & 41 & 42 & 43 & 44 \\
\hline Species & 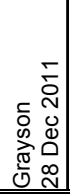 & 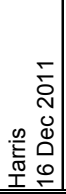 & 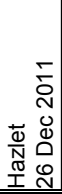 & 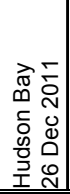 & 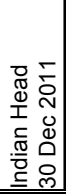 & 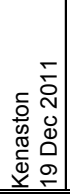 & 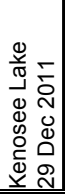 & 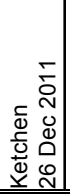 & 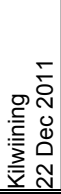 & 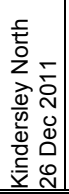 & 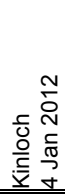 \\
\hline Canada Goose & 429 & & & & 750 & & & & & & \\
\hline Mallard & 117 & & & & 150 & & & & & & \\
\hline Common Goldeneye & 10 & & & & 12 & & & & & & \\
\hline Common Merganser & 3 & & & & & & & & & & \\
\hline Gray Partridge & & 9 & & (2) & 6 & & & & & & \\
\hline Ring-necked Pheasant & & & & & 1 & & & & & & \\
\hline Ruffed Grouse & 1 & & & & 2 & & & 7 & 1 & & 7 \\
\hline Sharp-tailed Grouse & 2 & 36 & 18 & 3 & 7 & & & & 7 & & \\
\hline Bald Eagle & 3 & & & (1) & 16 & & & & 2 & & (2) \\
\hline Northern Goshawk & & & & (1) & & & 1 & 1 & 1 & & (1) \\
\hline Rough-legged Hawk & & 3 & & & & & & & & & \\
\hline Golden Eagle & & & & & 2 & & & & & 1 & \\
\hline Merlin & & & & & 1 & & & & 1 & & \\
\hline Gyrfalcon & & & & & & & & & & & \\
\hline Rock Pigeon & & 5 & 9 & & 110 & 38 & 7 & 22 & 8 & 30 & \\
\hline Eurasian Collared-Dove & & & & & & & & & & & \\
\hline Mourning Dove & & & & & & & & & & & \\
\hline Great Horned Owl & 1 & 5 & 1 & & 2 & 2 & & & & & 1 \\
\hline Snowy Owl & & 6 & 3 & & 3 & 1 & & & & 2 & \\
\hline Downy Woodpecker & 2 & 1 & & 7 & 19 & & 4 & 3 & 2 & & 2 \\
\hline Hairy Woodpecker & 2 & & & 7 & 11 & & 3 & 3 & 3 & & 2 \\
\hline Northern Flicker & & & & & & & & & & & \\
\hline Pileated Woodpecker & & & & 1 & & & & & & & \\
\hline Northern Shrike & & & & & & & & (1) & 1 & & \\
\hline Gray Jay & & & & 17 & & & & & 4 & & 5 \\
\hline Blue Jay & 6 & & & 32 & 30 & (1) & 17 & 12 & 5 & & 23 \\
\hline Black-billed Magpie & 25 & 64 & 31 & 19 & 66 & 31 & 11 & 11 & 30 & 35 & 15 \\
\hline Common Raven & 48 & 18 & & 509 & 100 & 11 & 16 & 46 & 42 & 1 & 23 \\
\hline Horned Lark & & & 149 & & 20 & & & & & 6 & \\
\hline Black-capped Chickadee & 32 & 5 & & 87 & 122 & 2 & 71 & 8 & 34 & & 24 \\
\hline Boreal Chickadee & & & & 9 & & & & & 9 & & \\
\hline Red-breasted Nuthatch & & & & 2 & 5 & & 3 & & 1 & & 1 \\
\hline White-breasted Nuthatch & 1 & & & 8 & 12 & & 5 & 1 & 4 & & 7 \\
\hline Brown Creeper & & & & & & & 1 & & & & \\
\hline Golden-crowned Kinglet & & 1 & & & & & 1 & & & & \\
\hline American Robin & & 1 & & & & & & & & & \\
\hline European Starling & & 1 & & & 11 & & & 12 & & & \\
\hline Bohemian Waxwing & & 9 & & & 6 & (1) & & & & & \\
\hline Snow Bunting & 18 & & 588 & 10 & 215 & 3 & & 26 & 15 & 25 & 185 \\
\hline Dark-eyed Junco & & 10 & & & 7 & & 1 & & & & \\
\hline Pine Grosbeak & & 11 & & 111 & & & 7 & 31 & 82 & & 36 \\
\hline House Finch & & 2 & & & 12 & & & & & & \\
\hline White-winged Crossbill & & & & & & & & & 5 & & \\
\hline Common Redpoll & 152 & 46 & 103 & 139 & 176 & 4 & 83 & 69 & 128 & & 118 \\
\hline Hoary Redpoll & & 1 & 1 & & & & & 6 & & & 6 \\
\hline Pine Siskin & & & & & 4 & & & & 3 & & \\
\hline American Goldfinch & & & & & & & & & & & \\
\hline Evening Grosbeak & & & & 155 & & & & 1 & 67 & & 18 \\
\hline House Sparrow & 82 & 671 & 20 & 38 & 542 & 42 & 21 & 51 & 25 & 50 & 4 \\
\hline \begin{tabular}{|l} 
Total Birds Count Day \\
\end{tabular} & 935 & 905 & 923 & 1155 & 2423 & 134 & 252 & 312 & 488 & 150 & 487 \\
\hline Total Birds only in CP & 0 & 0 & 0 & 4 & 0 & 3 & 0 & 1 & 0 & 0 & 3 \\
\hline Total Species Count Day & 19 & 20 & 10 & 18 & 32 & 9 & 16 & 19 & 26 & 8 & 19 \\
\hline Total Species only in CP & 0 & 0 & 0 & 3 & 0 & 3 & 0 & 1 & 0 & 0 & 2 \\
\hline
\end{tabular}


Table 3-5. Species found on 8 or more counts; ( ) = seen during Count Period (CP).

\begin{tabular}{|c|c|c|c|c|c|c|c|c|c|c|c|}
\hline Map Number & 45 & 46 & 47 & 48 & 49 & 50 & 51 & 52 & 53 & 54 & 55 \\
\hline Species & 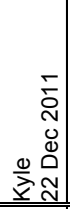 & 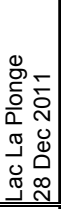 & 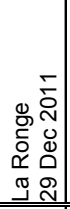 & 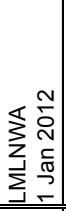 & 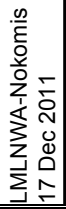 & 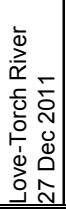 & 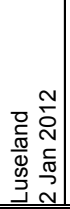 & 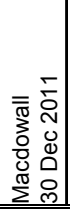 & 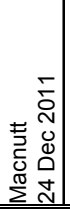 & 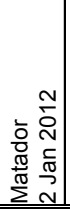 & 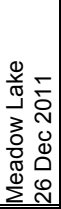 \\
\hline \multicolumn{12}{|l|}{ Canada Goose } \\
\hline Mallard & & & 1 & & & & & & & & \\
\hline Common Goldeneye & & & 1 & & & & & & & & \\
\hline Common Merganser & & & 1 & & & & & & & & \\
\hline Gray Partridge & 12 & & & & & (11) & 7 & & & & \\
\hline \multicolumn{12}{|l|}{ Ring-necked Pheasant } \\
\hline Ruffed Grouse & & 1 & & & & 3 & & 2 & 4 & & (2) \\
\hline Sharp-tailed Grouse & 60 & & & 2 & 11 & & (2) & 3 & & & \\
\hline Bald Eagle & & & & & & (1) & & (1) & (1) & & \\
\hline Northern Goshawk & (1) & & & & & & & (1) & (1) & & \\
\hline Rough-legged Hawk & (1) & & & & 1 & & & & & 1 & \\
\hline \multicolumn{12}{|l|}{ Golden Eagle } \\
\hline Merlin & 1 & & & & & & & & & & \\
\hline Gyrfalcon & (1) & & & & & & & & (1) & (1) & \\
\hline Rock Pigeon & 238 & & & 41 & 18 & 32 & 152 & & & & 17 \\
\hline Eurasian Collared-Dove & (2) & & & & & & 9 & & & & \\
\hline \multicolumn{12}{|l|}{ Mourning Dove } \\
\hline Great Horned Owl & 5 & & & 5 & 4 & & 1 & (1) & & & \\
\hline Snowy Owl & 11 & & & 11 & 5 & & 1 & & 1 & 2 & \\
\hline Downy Woodpecker & 2 & 2 & 2 & & & 11 & & 7 & & & \\
\hline Hairy Woodpecker & & 3 & (1) & & (1) & 11 & & 6 & 5 & & 1 \\
\hline \multicolumn{12}{|l|}{ Northern Flicker } \\
\hline Pileated Woodpecker & & & & & & (1) & & 2 & & & \\
\hline Northern Shrike & & & & & & & & (1) & & & \\
\hline Gray Jay & & 9 & 5 & & & 18 & & & & & 3 \\
\hline Blue Jay & 2 & 5 & & & & 18 & 13 & 7 & 10 & & 5 \\
\hline Black-billed Magpie & 75 & 20 & & 22 & 30 & 86 & 122 & 22 & 25 & 5 & 13 \\
\hline Common Raven & 5 & 117 & 42 & 1 & 5 & 152 & 9 & 26 & 39 & & 68 \\
\hline Horned Lark & $(2)$ & & & & & & 22 & & & & \\
\hline Black-capped Chickadee & 1 & 15 & 3 & 2 & 2 & 119 & 10 & 69 & 68 & & 10 \\
\hline Boreal Chickadee & & 4 & & & & 8 & & & & & $(1)$ \\
\hline Red-breasted Nuthatch & & 2 & & & & 10 & 1 & 1 & & & 4 \\
\hline White-breasted Nuthatch & & & & & & 4 & & 6 & 1 & & $(1)$ \\
\hline \multicolumn{12}{|l|}{ Brown Creeper } \\
\hline \multicolumn{12}{|l|}{ Golden-crowned Kinglet } \\
\hline \multicolumn{12}{|l|}{ American Robin } \\
\hline European Starling & & & & 25 & & 42 & 9 & & & & \\
\hline Bohemian Waxwing & (15) & & 117 & & 1 & 81 & 149 & $(25)$ & & & \\
\hline Snow Bunting & 350 & & & 988 & 720 & 2011 & 330 & 315 & 124 & 564 & \\
\hline Dark-eyed Junco & & & (1) & 5 & & 3 & & & & & \\
\hline Pine Grosbeak & 4 & 18 & 8 & 1 & & 191 & 32 & 72 & 23 & & 21 \\
\hline House Finch & & & & 5 & & & 10 & & & & 1 \\
\hline \multicolumn{12}{|l|}{ White-winged Crossbill } \\
\hline Common Redpoll & 175 & 21 & 43 & 53 & 140 & 1590 & 131 & 316 & 22 & & \\
\hline Hoary Redpoll & & 20 & & & & 5 & & 3 & & & 1 \\
\hline Pine Siskin & & & & & & 10 & & & 6 & & \\
\hline \multicolumn{12}{|l|}{ American Goldfinch } \\
\hline Evening Grosbeak & & 11 & 26 & & & 145 & & 71 & & & 4 \\
\hline House Sparrow & 836 & & & 339 & 110 & 152 & 272 & 2 & 34 & 23 & 6 \\
\hline Total Birds Count Day & 1780 & 261 & 249 & 1500 & 1048 & 4704 & 1280 & 930 & 364 & 595 & 154 \\
\hline Total Birds only in CP & 22 & 0 & 2 & 0 & 1 & 13 & 3 & 32 & 3 & 1 & 4 \\
\hline Total Species Count Day & 17 & 15 & 11 & 14 & 13 & 24 & 18 & 17 & 15 & 5 & 13 \\
\hline Total Species only in CP & 6 & 0 & 2 & 0 & & 3 & 2 & 8 & 3 & 1 & \\
\hline
\end{tabular}


Table 3-6. Species found on 8 or more counts; ()$=$ seen during Count Period (CP).

\begin{tabular}{|c|c|c|c|c|c|c|c|c|c|c|c|}
\hline Map Number & 56 & 57 & 58 & 59 & 60 & 61 & 62 & 63 & 64 & 65 & 66 \\
\hline Species & 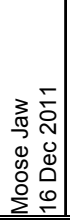 & 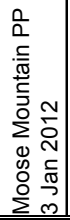 & 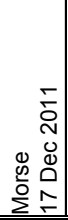 & 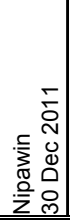 & 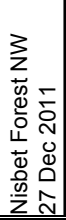 & 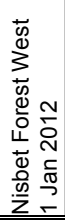 & 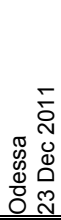 & 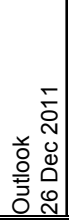 & 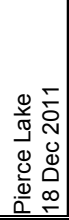 & 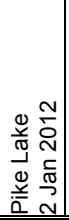 & 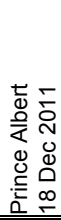 \\
\hline Canada Goose & 38 & & & & & & & $(75)$ & & & \\
\hline Mallard & 37 & & & & & & & & 1 & & \\
\hline Common Goldeneye & & & & & & & & (14) & 43 & & \\
\hline \multicolumn{12}{|l|}{ Common Merganser } \\
\hline Gray Partridge & 3 & & 105 & 4 & & & & & & & \\
\hline Ring-necked Pheasant & & & 11 & & & & & & & & \\
\hline Ruffed Grouse & & & & 2 & $(1)$ & & 3 & & & 1 & 4 \\
\hline Sharp-tailed Grouse & & 2 & 18 & & & & 15 & & & 8 & \\
\hline Bald Eagle & & & 1 & 2 & & & 2 & 4 & 1 & 7 & 1 \\
\hline \multicolumn{12}{|l|}{ Northern Goshawk } \\
\hline Rough-legged Hawk & & & & & & & & & & 1 & \\
\hline Golden Eagle & & & 1 & & & & & & & & \\
\hline Merlin & 1 & & 1 & & & & & & & & 1 \\
\hline Gyrfalcon & & & 1 & (1) & & & & & & & \\
\hline Rock Pigeon & 401 & 9 & 278 & 128 & & & & 2 & & 76 & 467 \\
\hline Eurasian Collared-Dove & 2 & & 6 & & & & & 20 & & & \\
\hline Mourning Dove & & & 2 & & & & & & & (2) & \\
\hline Great Horned Owl & 1 & & 19 & & & & 1 & 1 & & 6 & \\
\hline Snowy Owl & 6 & 1 & 17 & & & & 2 & & & 1 & \\
\hline Downy Woodpecker & 1 & & & 9 & 3 & 4 & 4 & 1 & 1 & 27 & 5 \\
\hline Hairy Woodpecker & 1 & 1 & & 4 & 1 & 3 & 6 & 1 & & 22 & 6 \\
\hline \begin{tabular}{|l} 
Northern Flicker \\
\end{tabular} & 1 & & & & & & 4 & & & & \\
\hline Pileated Woodpecker & & & & 1 & & & 2 & & & 2 & \\
\hline Northern Shrike & & & 1 & & & & & & & 3 & \\
\hline Gray Jay & & & & 2 & & & & & 2 & & 1 \\
\hline Blue Jay & & 1 & & 30 & 7 & 10 & 2 & 5 & 3 & 28 & 21 \\
\hline Black-billed Magpie & 23 & 24 & 71 & 58 & 3 & 12 & 25 & 68 & 7 & 144 & 116 \\
\hline Common Raven & 3 & 121 & 7 & 291 & 1 & 23 & 12 & 23 & 35 & 174 & 409 \\
\hline Horned Lark & & & 67 & & & & & & & & \\
\hline Black-capped Chickadee & 14 & 16 & & 99 & 51 & 25 & 20 & 5 & 2 & 425 & 189 \\
\hline Boreal Chickadee & & & & & & & & & & & 2 \\
\hline Red-breasted Nuthatch & 2 & & & 18 & & & & 1 & & 3 & 1 \\
\hline White-breasted Nuthatch & 3 & 6 & & 2 & 3 & 1 & & (2) & & 8 & 8 \\
\hline \multicolumn{12}{|l|}{ Brown Creeper } \\
\hline \multicolumn{12}{|l|}{ Golden-crowned Kinglet } \\
\hline American Robin & $(2)$ & & & & & & 2 & & & 10 & 2 \\
\hline European Starling & 101 & & 28 & 210 & & & & & & 822 & 5 \\
\hline Bohemian Waxwing & & & 1 & 561 & & & & & & 205 & 1153 \\
\hline Snow Bunting & & & 1473 & & & 30 & 40 & & & 878 & 12 \\
\hline Dark-eyed Junco & 6 & & & $(1)$ & & & & & & 7 & \\
\hline Pine Grosbeak & & & & 116 & 5 & 36 & & $(4)$ & 5 & 131 & 83 \\
\hline House Finch & (1) & & 1 & & & & & & & 4 & \\
\hline White-winged Crossbill & & & & 5 & & & & & & & 2 \\
\hline Common Redpoll & 3 & 59 & 506 & 446 & 12 & 108 & & & 22 & 591 & 231 \\
\hline Hoary Redpoll & & & & 4 & & 1 & & & & 7 & 5 \\
\hline Pine Siskin & & & & 1 & & & & & & & 42 \\
\hline \multicolumn{12}{|l|}{ American Goldfinch } \\
\hline Evening Grosbeak & & & & 69 & (6) & 47 & & & & & 17 \\
\hline House Sparrow & 80 & 42 & 1235 & 231 & & & & 149 & & 376 & 362 \\
\hline \begin{tabular}{|l} 
Total Birds Count Day \\
\end{tabular} & 731 & 282 & 3851 & 2294 & 86 & 300 & 140 & 280 & 122 & 3967 & 3154 \\
\hline Total Birds only in CP & 4 & 0 & 1 & 2 & 9 & 1 & 0 & 95 & 0 & 2 & 2 \\
\hline \begin{tabular}{|l|l} 
Total Species Count Day \\
\end{tabular} & 22 & 11 & 23 & 24 & 9 & 12 & 15 & 12 & 11 & 27 & 28 \\
\hline Total Species only in CP & 3 & 0 & 1 & 2 & 4 & 1 & 0 & 4 & 0 & 1 & 1 \\
\hline
\end{tabular}


Table 3-7. Species found on 8 or more counts; ()$=$ seen during Count Period (CP).

\begin{tabular}{|c|c|c|c|c|c|c|c|c|c|c|c|}
\hline Map Number & 67 & 68 & 69 & 70 & 71 & 72 & 73 & 74 & 75 & 76 & 77 \\
\hline Species & 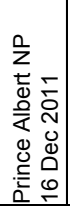 & 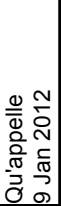 & 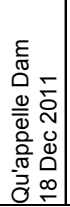 & 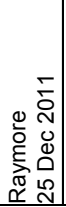 & 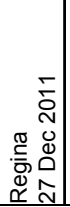 & 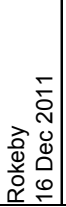 & 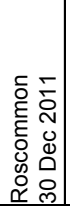 & 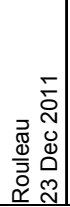 & 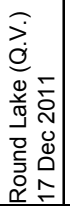 & 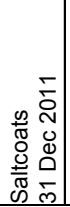 & 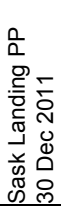 \\
\hline Canada Goose & & & 2503 & & 379 & & & & & & \\
\hline Mallard & & & 1101 & & 3 & & & & 18 & & 1 \\
\hline Common Goldeneye & & & 54 & & & & & & 1 & & \\
\hline Common Merganser & & & 1 & & & & & & 1 & & \\
\hline Gray Partridge & & & 3 & & 93 & 33 & & & & & 15 \\
\hline Ring-necked Pheasant & & & & & & & & & & & 1 \\
\hline Ruffed Grouse & 7 & & & & & 1 & 7 & & & 2 & \\
\hline Sharp-tailed Grouse & & & 26 & & 11 & 12 & & & & 15 & 31 \\
\hline Bald Eagle & & & 10 & & & 1 & & & 2 & & \\
\hline Northern Goshawk & & & 1 & & & & (1) & & & & \\
\hline Rough-legged Hawk & & & & & 1 & & & & & & \\
\hline Golden Eagle & & & 2 & & & & & & 3 & (1) & 3 \\
\hline Merlin & & & & & 4 & & & & & & \\
\hline Gyrfalcon & & & 1 & & & & & & & & 1 \\
\hline Rock Pigeon & & & 41 & 4 & 587 & 50 & & 69 & 106 & & 61 \\
\hline Eurasian Collared-Dove & & & 35 & & & & & & & & \\
\hline \multicolumn{12}{|l|}{ Mourning Dove } \\
\hline Great Horned Owl & & & 3 & 1 & 3 & 1 & (1) & 4 & & & 3 \\
\hline Snowy Owl & & & 3 & & 45 & 1 & & 19 & & 1 & 4 \\
\hline Downy Woodpecker & 2 & 3 & 1 & 5 & 13 & 3 & 4 & & 2 & 8 & \\
\hline Hairy Woodpecker & 5 & 4 & 7 & & 4 & 1 & 6 & & 4 & 11 & \\
\hline Northern Flicker & & & 2 & & 6 & & & & & & \\
\hline Pileated Woodpecker & 3 & & & & & & (1) & & & & \\
\hline Northern Shrike & & & 1 & & & & & & & & \\
\hline Gray Jay & 16 & & & & & & & & & & \\
\hline Blue Jay & 1 & & 4 & & 12 & & 8 & & 33 & 8 & 1 \\
\hline Black-billed Magpie & 9 & 4 & 85 & 16 & 39 & 37 & 14 & 2 & 31 & 39 & 68 \\
\hline Common Raven & 30 & 3 & 6 & 31 & 63 & 24 & 8 & & 14 & 85 & 2 \\
\hline Horned Lark & & & 1 & & & & & & & & 2 \\
\hline Black-capped Chickadee & 22 & 14 & 40 & 19 & 96 & 25 & 48 & & 22 & 49 & 1 \\
\hline Boreal Chickadee & 146 & & & & & & & & & & \\
\hline Red-breasted Nuthatch & 1. & & 2 & & 40 & & & & 1 & & 1 \\
\hline White-breasted Nuthatch & & & & & 9 & & 1 & & 9 & 7 & \\
\hline Brown Creeper & & & & & 4 & & & & 1 & & \\
\hline Golden-crowned Kinglet & & & & & 4 & & & & & & \\
\hline American Robin & & & & & 12 & & 1 & & 1 & & \\
\hline European Starling & & & 7 & & 208 & & & 30 & & & 3 \\
\hline Bohemian Waxwing & & & 117 & 205 & & & 61 & & & & \\
\hline Snow Bunting & & & 162 & 1 & 900 & 160 & $(500)$ & & & 125 & \\
\hline Dark-eyed Junco & & & & (1) & 34 & & 3 & & & & \\
\hline Pine Grosbeak & & & 46 & 4 & 14 & 15 & 58 & & 11 & 12 & \\
\hline House Finch & & & 18 & 5 & 89 & & & & & & \\
\hline White-winged Crossbill & 25 & & & & & & $(40)$ & & & & \\
\hline Common Redpoll & 2 & & 282 & 31 & 44 & 17 & 93 & & 484 & 127 & 39 \\
\hline Hoary Redpoll & & & & 1 & & & 4 & & 1 & & \\
\hline Pine Siskin & 3 & & & & 24 & & & & & & \\
\hline \multicolumn{12}{|l|}{ American Goldfinch } \\
\hline Evening Grosbeak & & & & & & & & & & 2 & \\
\hline House Sparrow & & 18 & 684 & 126 & 1286 & 222 & 36 & 80 & 111 & 54 & 402 \\
\hline Total Birds Count Day & 277 & 46 & 5378 & 471 & 4059 & 604 & 352 & 204 & 856 & 545 & 640 \\
\hline Total Birds only in CP & 0 & 0 & 0 & 1 & 3 & 0 & 543 & 0 & 0 & 1 & 1 \\
\hline Total Species Count Day & 18 & 6 & 37 & 14 & 34 & 17 & 15 & 6 & 20 & 15 & 19 \\
\hline Total Species only in CP & 0 & 0 & 0 & 1 & 2 & 0 & 5 & 0 & 0 & 1 & 1 \\
\hline
\end{tabular}


Table 3-8. Species found on 8 or more counts; ()$=$ seen during Count Period $(C P)$.

\begin{tabular}{|c|c|c|c|c|c|c|c|c|c|c|c|}
\hline Map Number & 78 & 79 & 80 & 81 & 82 & 83 & 84 & 85 & 86 & 87 & 88 \\
\hline Species & 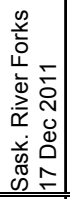 & 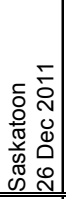 & 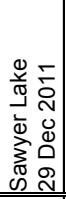 & 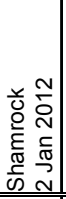 & 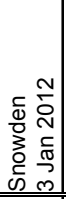 & 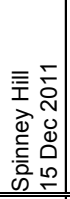 & 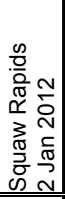 & 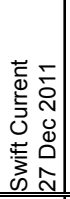 & 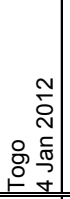 & 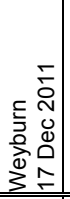 & 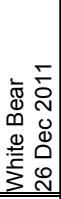 \\
\hline Canada Goose & & 421 & & & & & & & & & \\
\hline Mallard & & 84 & & & & & & & & & \\
\hline Common Goldeneye & & 181 & & & & & 268 & & & & \\
\hline Common Merganser & & 2 & & & & & 2 & & & & \\
\hline Gray Partridge & & 20 & & 11 & & & & 41 & & 20 & \\
\hline Ring-necked Pheasant & & & & 1 & & & & 11 & & 19 & \\
\hline Ruffed Grouse & 1 & 1 & 5 & & & & 2 & & 2 & & \\
\hline Sharp-tailed Grouse & & & & & 10 & & 5 & (25) & 4 & & 8 \\
\hline Bald Eagle & & 4 & & & & & 3 & (1) & & & \\
\hline Northern Goshawk & & & (1) & & & & & & & & \\
\hline Rough-legged Hawk & & 1 & & & & & & (1) & & & 1 \\
\hline Golden Eagle & & & & & & & & & & 1 & \\
\hline Merlin & & 11 & & & & & & & 1 & 1 & \\
\hline Gyrfalcon & & & & & & & & & & & \\
\hline Rock Pigeon & & 2534 & & 102 & & 29 & & 174 & 6 & 210 & 92 \\
\hline Eurasian Collared-Dove & & 8 & & & & & & 46 & & 15 & \\
\hline Mourning Dove & & 1 & & 3 & & & & & & 3 & \\
\hline Great Horned Owl & & 3 & & 11 & & & & 2 & & 1 & 1 \\
\hline Snowy Owl & & & & 14 & & & & 9 & 1 & 1 & 3 \\
\hline Downy Woodpecker & 3 & 16 & 1 & & 11 & 3 & & 1 & 15 & 1 & \\
\hline Hairy Woodpecker & 6 & 8 & 1 & & 6 & 3 & & 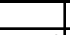 & 10 & & \\
\hline Northern Flicker & & 11 & & & & 1 & & 4 & & & \\
\hline Pileated Woodpecker & 2 & & (1) & & & 1 & & & 1 & & \\
\hline Northern Shrike & & 3 & & & & & & 1 & & & \\
\hline Gray Jay & 4 & & 2 & & 3 & & 11 & & & & \\
\hline Blue Jay & 10 & 60 & 2 & & 16 & 5 & 8 & & 68 & & \\
\hline Black-billed Magpie & 32 & 410 & 14 & 29 & 84 & 40 & 15 & 154 & 12 & 22 & 24 \\
\hline Common Raven & 25 & 313 & 19 & & 83 & 25 & 27 & 2 & 118 & & 1 \\
\hline Horned Lark & & & & & & 2 & & 3 & & & \\
\hline Black-capped Chickadee & 34 & 713 & 14 & & 87 & 27 & 22 & 2 & 78 & & \\
\hline Boreal Chickadee & 8 & & & & 9 & & 5 & & 10 & & \\
\hline Red-breasted Nuthatch & & 76 & & & 7 & & 15 & 18 & & 4 & \\
\hline White-breasted Nuthatch & 1 & 2 & & & 6 & & & 3 & 6 & 1 & \\
\hline Brown Creeper & & 1 & & & & & 1 & & & & \\
\hline Golden-crowned Kinglet & 1 & 8 & & & & & & 2 & & & \\
\hline American Robin & & 1 & & & & 1 & & & & & \\
\hline European Starling & & 81 & 16 & & & 13 & 1 & & & 400 & 3 \\
\hline Bohemian Waxwing & & 298 & & & & & & 10 & & 4 & \\
\hline Snow Bunting & 5 & & 350 & 928 & 160 & 3 & 326 & 305 & & & \\
\hline Dark-eyed Junco & & 13 & & & & & & 9 & & 7 & \\
\hline Pine Grosbeak & 42 & 12 & 14 & & 110 & 9 & 18 & 8 & 61 & & \\
\hline House Finch & & 430 & & 2 & & 5 & & 79 & & & \\
\hline White-winged Crossbill & & 11 & & & & & & 12 & & & \\
\hline Common Redpoll & 372 & 439 & 34 & 54 & 518 & 274 & 187 & 60 & 153 & 12 & \\
\hline Hoary Redpoll & 3 & 3 & & 1 & & & 1 & & & & \\
\hline Pine Siskin & & 3 & & & & & & 7 & & & \\
\hline American Goldfinch & & (1) & & & & & & & & & \\
\hline Evening Grosbeak & & 2 & 30 & & 147 & 4 & & & 77 & & \\
\hline House Sparrow & 28 & 2513 & 120 & 675 & 2 & 200 & 13 & 618 & \begin{tabular}{l|l}
31 \\
\end{tabular} & 542 & 35 \\
\hline Total Birds Count Day & 577 & 8774 & 622 & 1834 & 1275 & 652 & 940 & 1584 & 655 & 1264 & 168 \\
\hline Total Birds only in CP & 0 & 2 & 4 & 0 & 0 & 0 & 2 & 27 & 0 & 0 & 0 \\
\hline Total Species Count Day & 17 & 48 & 14 & 15 & 17 & 19 & 25 & 28 & 19 & 18 & 9 \\
\hline Total Species only in CP & 0 & 2 & 3 & 0 & 0 & 0 & 2 & 3 & 0 & 0 & 0 \\
\hline
\end{tabular}


Table 3-9. Species found on 8 or more counts; () = seen during Count Period (CP).

\begin{tabular}{|c|c|c|c|c|c|c|c|}
\hline Map Number & 89 & 90 & 91 & & & & \\
\hline Species & 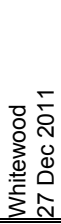 & 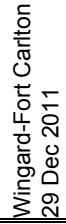 & 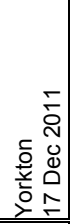 & 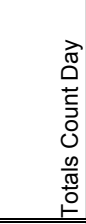 & 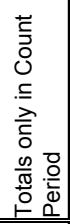 & 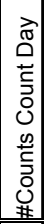 & 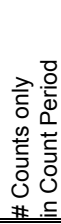 \\
\hline Canada Goose & & & & 27330 & 75 & 12 & 1 \\
\hline Mallard & & & 3 & 11335 & 1 & 20 & 1 \\
\hline Common Goldeneye & & & & 841 & 14 & 14 & 1 \\
\hline Common Merganser & & & & 97 & 0 & 9 & 0 \\
\hline Gray Partridge & 2 & 2 & & 482 & 30 & 25 & 4 \\
\hline Ring-necked Pheasant & & & & 227 & 0 & 11 & 0 \\
\hline Ruffed Grouse & (1) & 3 & 1 & 83 & 11 & 32 & 7 \\
\hline Sharp-tailed Grouse & (6) & 21 & & 799 & 35 & 42 & 4 \\
\hline Bald Eagle & 2 & & 1 & 119 & 10 & 26 & 8 \\
\hline Northern Goshawk & & & & 10 & 8 & 8 & 8 \\
\hline Rough-legged Hawk & & 1 & & 19 & 2 & 13 & 2 \\
\hline Golden Eagle & & & & 28 & 1 & 17 & 1 \\
\hline Merlin & 1 & & & 26 & 1 & 13 & 1 \\
\hline Gyrfalcon & & & & 3 & 5 & 3 & 5 \\
\hline Rock Pigeon & 38 & & 159 & 8384 & 0 & 61 & 0 \\
\hline Eurasian Collared-Dove & 5 & & 7 & 333 & 2 & 17 & 1 \\
\hline Mourning Dove & & & & 11 & 3 & 6 & 2 \\
\hline Great Horned Owl & (1) & (1) & 1 & 115 & 7 & 37 & 7 \\
\hline Snowy Owl & 2 & & & 233 & 1 & 40 & 1 \\
\hline Downy Woodpecker & 14 & 2 & 17 & 334 & 2 & 65 & 2 \\
\hline Hairy Woodpecker & 8 & 4 & 5 & 297 & 2 & 65 & 2 \\
\hline Northern Flicker & & & & 37 & 1 & 12 & 1 \\
\hline Pileated Woodpecker & (1) & 1 & 1 & 22 & 7 & 13 & 7 \\
\hline Northern Shrike & & 1 & & 20 & 2 & 13 & 2 \\
\hline Gray Jay & & 2 & & 114 & 2 & 21 & 1 \\
\hline Blue Jay & 22 & 15 & 4 & 736 & 4 & 61 & 2 \\
\hline Black-billed Magpie & 43 & 19 & 13 & 4243 & 0 & 89 & 0 \\
\hline Common Raven & 94 & 49 & 149 & 5392 & 1 & 82 & 1 \\
\hline Horned Lark & & & & 325 & 2 & 12 & 1 \\
\hline Black-capped Chickadee & 182 & 10 & 66 & 4287 & 0 & 80 & 0 \\
\hline Boreal Chickadee & & & & 226 & 1 & 13 & 1 \\
\hline Red-breasted Nuthatch & & & 1 & 285 & 0 & 39 & 0 \\
\hline White-breasted Nuthatch & 15 & 4 & 7 & 223 & 3 & 51 & 2 \\
\hline Brown Creeper & 2 & & & 14 & 1 & 9 & 1 \\
\hline Golden-crowned Kinglet & & & & 20 & 0 & 8 & 0 \\
\hline American Robin & & & & 34 & 6 & 11 & 5 \\
\hline European Starling & & & 81 & 2619 & 4 & 35 & 1 \\
\hline Bohemian Waxwing & 70 & 50 & 30 & 4143 & 65 & 32 & 5 \\
\hline Snow Bunting & 845 & 10 & & 14789 & 708 & 51 & 5 \\
\hline Dark-eyed Junco & 1 & & 1 & 126 & 3 & 20 & 3 \\
\hline Pine Grosbeak & 8 & 42 & 19 & 2033 & 6 & 59 & 2 \\
\hline House Finch & 3 & & & 779 & 6 & 21 & 3 \\
\hline White-winged Crossbill & & & & 85 & 41 & 8 & 2 \\
\hline Common Redpoll & 803 & 64 & 54 & 15280 & 0 & 81 & 0 \\
\hline Hoary Redpoll & 3 & 1 & & 110 & 0 & 32 & 0 \\
\hline Pine Siskin & & & & 103 & 0 & 10 & 0 \\
\hline American Goldfinch & (1) & 1 & & 5 & 17 & 3 & 6 \\
\hline Evening Grosbeak & & 23 & & 1190 & 6 & 27 & 1 \\
\hline House Sparrow & 284 & 54 & 530 & 19550 & 0 & 79 & 0 \\
\hline Total Birds Count Day & 2447 & 380 & 1150 & 128510 & 1121 & & \\
\hline Total Birds only in CP & 10 & 1 & 1 & 1121 & & & \\
\hline Total Species Count Day & 22 & 23 & 21 & & & & \\
\hline Total Species only in CP & 5 & 1 & 1 & & & & \\
\hline
\end{tabular}


Table 4-1. Species found in fewer than 8 counts.

\begin{tabular}{|c|c|}
\hline Species & Locality and Number $\left({ }^{*}=\right.$ Count Period $)$ \\
\hline $\begin{array}{l}\text { Greater White-fronted } \\
\text { Goose }\end{array}$ & Coronach (2) \\
\hline Snow Goose & Indian Head (1), Moose Jaw (1), Regina (1) \\
\hline Cackling Goose & Estevan (5), Gardiner Dam (50), Qu'Appelle Dam (3) \\
\hline Gadwall & Crooked Lake (2), Estevan (3) \\
\hline Northern Pintail & Estevan (1) \\
\hline Green-winged Teal & Estevan (1) \\
\hline Canvasback & Estevan (2), Saskatoon (1), Squaw Rapids $\left(1^{*}\right)$ \\
\hline Redhead & Gardiner Dam (37) \\
\hline Ring-necked Duck & Crooked Lake (2), , Estevan (7), Grayson (1) \\
\hline Lesser Scaup & $\begin{array}{l}\text { Crooked Lake (1), Estevan (8), Fort Qu'Appelle (1), } \\
\text { Gardiner Dam (21), Qu'Appelle Dam (2), Saskatoon (2), } \\
\text { Squaw Rapids (1) }\end{array}$ \\
\hline White-winged Scoter & Gardiner Dam (2) \\
\hline Long-tailed Duck & Squaw Rapids (1) \\
\hline Bufflehead & Fort Qu'Appelle (1), Saskatoon (1) \\
\hline Barrow's Goldeneye & Squaw Rapids $\left(1^{*}\right)$ \\
\hline Hooded Merganser & Estevan (5), Gardiner Dam (2), Squaw Rapids (2) \\
\hline Red-breasted Merganser & Coronach (1) \\
\hline Ruddy Duck & Estevan (1), Fort Qu'Appelle $\left(1^{*}\right)$ \\
\hline Spruce Grouse & Hudson Bay (1), Love-Torch River (1) \\
\hline Willow Ptarmigan & Creighton (20), Lac La Plonge (13) \\
\hline Wild Turkey & Broadview (4), Cypress Hills P.P.(2) \\
\hline Pied-billed Grebe & Coronach (1), Estevan (2) \\
\hline Horned Grebe & Gardiner Dam (1) \\
\hline Red-necked Grebe & Gardiner Dam (1) \\
\hline Western Grebe & Coronach (1) \\
\hline American White Pelican & Fort Qu'Appelle (1), Gardiner Dam (5) \\
\hline Turkey Vulture & Moose Jaw $\left(1^{*}\right)$ \\
\hline Northern Harrier & Archerwill (1*), Grasslands N. P. (1) \\
\hline Sharp-shinned Hawk & $\begin{array}{l}\text { Clark's Crossing (1), Qu'Appelle Dam (1), Saskatoon (2), } \\
\text { Swift Current (1) }\end{array}$ \\
\hline Cooper's Hawk & MacDowall $\left({ }^{*}\right)$, Regina $\left(1^{*}\right)$ \\
\hline Red-tailed Hawk & Gardiner Dam (1), Regina $\left(1^{*}\right)$, Saskatoon (2) \\
\hline American Kestrel & Morse $\left(1^{\star}\right)$ \\
\hline Prairie Falcon & $\begin{array}{l}\text { Kyle (2), Qu'Appelle Dam (1), Saskatchewan Landing P.P. } \\
\left(1^{*}\right) \text {, Shamrock (1) }\end{array}$ \\
\hline
\end{tabular}


Table 4-1. (Con't) Species found in fewer than 8 counts.

\begin{tabular}{|l|l|}
\hline American Coot & Coronach (2), Estevan (7), Fort Qu'Appelle (17) \\
\hline Killdeer & Gardiner Dam (1) \\
\hline Herring Gull & Gardiner Dam (7) \\
\hline Northern Hawk Owl & $\begin{array}{l}\text { Kenaston }\left(1^{*}\right), \text { Kinloch (1), Nipawin (1), Nisbet Forest West (1*), } \\
\text { Prince Albert (3), Sawyer Lake }\left(2^{*}\right), \text { Squaw Rapids (2) }\end{array}$ \\
\hline
\end{tabular}

Table 4-2. Species found in fewer than 8 counts.

\begin{tabular}{|c|c|}
\hline Species & Locality and Number $\left({ }^{*}=\right.$ Count Period $)$ \\
\hline Great Gray Owl & Candle Lake (2), Prince Albert NP (1) \\
\hline Short-eared Owl & $\begin{array}{l}\text { Biggar (2), Clark's Crossing (2), Grassland NP (2), Rokeby } \\
\text { (1) }\end{array}$ \\
\hline Northern Saw-whet Owl & Prince Albert N.P. (1) \\
\hline $\begin{array}{l}\text { American Three-toed } \\
\text { Woodpecker }\end{array}$ & $\begin{array}{l}\text { Kilwinning (2), Nisbet Forest Northwest }\left(1^{*}\right) \text {, Prince Albert } \\
\text { (1), Squaw Rapids (1) }\end{array}$ \\
\hline $\begin{array}{l}\text { Black-backed } \\
\text { Woodpecker }\end{array}$ & $\begin{array}{l}\text { MacDowall }\left(1^{*}\right) \text {, Nisbet Forest Northwest }\left(1^{*}\right) \text {, Prince } \\
\text { Albert N.P. (1), Squaw Rapids (3) }\end{array}$ \\
\hline American Crow & Craven (1), Regina (8), Saskatoon (16), Swift Current (1) \\
\hline Winter Wren & Fort Qu'Appelle (1) \\
\hline Townsend's Solitaire & $\begin{array}{l}\text { Biggar (1), Clark's Crossing (1), Craven (1), Cypress Hills } \\
\text { P.P. (1), Regina }\left(1^{*}\right) \text {, Saskatoon (3), Swift Current (1) }\end{array}$ \\
\hline Varied Thrush & Qu'Appelle Dam (1) \\
\hline Cedar Waxwing & $\begin{array}{l}\text { Fort Qu'Appelle (1), Raymore (20), Regina (20), Saskatoon } \\
\text { (9), Snowden (16) }\end{array}$ \\
\hline Orange-crowned Warbler & Togo (1) \\
\hline American Tree Sparrow & Qu'Appelle Dam (1) \\
\hline White-throated Sparrow & Ketchen (1), Saskatoon (2) \\
\hline Harris's Sparrow & Kyle (1), LMLNWA-Nokomis (1), Morse (1), Saskatoon (1) \\
\hline White-crowned Sparrow & Ketchen (1) \\
\hline Northern Cardinal & Prince Albert $\left(2^{*}\right)$, Yorkton (1) \\
\hline Red-winged Blackbird & Eastend (1), MacNutt (1) \\
\hline Yellow-headed Blackbird & Good Spirit Lake (1), Indian Head (2) \\
\hline Rusty Blackbird & $\begin{array}{l}\text { Balgonie (3), Clark's Crossing (1), Kilwinning (6), } \\
\text { Saskatchewan Landing PP (1), Spinney Hill (7) }\end{array}$ \\
\hline Common Grackle & $\begin{array}{l}\text { Churchbridge (2), Estevan }\left(1^{*}\right) \text {, Moose Jaw (3), Regina } \\
(2) \text {, Saskatoon }\left(1^{*}\right) \text {, Shamrock (1) }\end{array}$ \\
\hline $\begin{array}{l}\text { Gray-crowned Rosy- } \\
\text { Finch }\end{array}$ & Cabri (1), Wingard-Fort Carlton \\
\hline Purple Finch & Prince Albert (5) \\
\hline Red Crossbill & Cypress Hills PP (2), Prince Albert NP (2), Saskatoon (22) \\
\hline
\end{tabular}


Table 5. Birds not identified to species.

\begin{tabular}{|l|l|}
\hline Category & Locality and Number (*=Count Period) \\
\hline Duck sp. & Qu'Appelle Dam (120), Saskatoon (3) \\
\hline Grebe sp. & Coronach (1) \\
\hline Small Accipiter & Avonlea (1*), \\
\hline Accipiter sp. & Regina (1) \\
\hline Eagle sp. & Shamrock (1) \\
\hline Falcon sp. & Love-Torch River (1) \\
\hline Woodpecker sp. & Clark's Crossing (1), Raymore (2), Saskatoon (1) \\
\hline Crossbill sp. & Kinloch (9) \\
\hline Finch sp. & Saskatoon (11) \\
\hline
\end{tabular}

Figure 1. Location of 2011 counts (numbers correspond to those in text under Count areas and participants).

Natural Vegetation Zones*
A. Subarctic Woodland
B. Northern Boreal Forest
C. Southern Boreal Forest
D. Aspen Parkland
E. Mixed Prairie
F. Dry Mixed Prairie
G. Cypress Hills

*adapted from: Thorpe, J. 1999.

Natural Vegetation. P. 133 in

Atlas of Saskatchewan (K. Fung, ed). Univ. of Sask., Saskatoon.
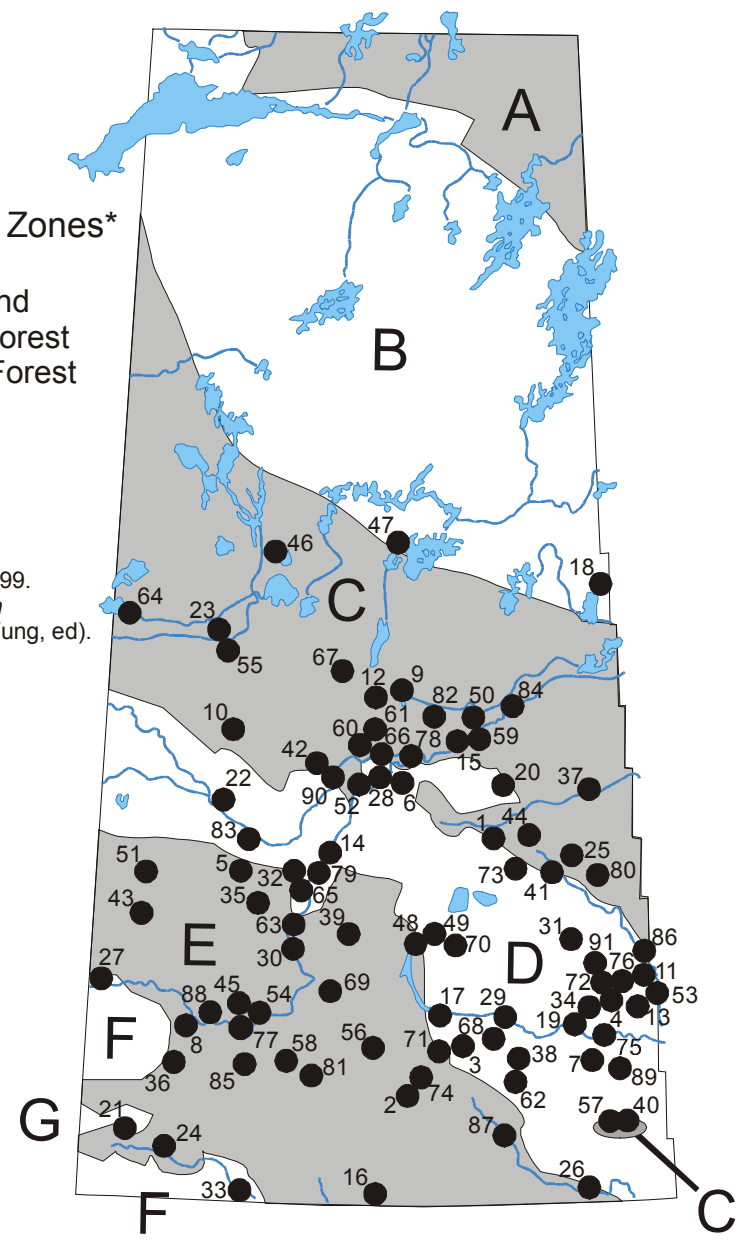

70 (1) March 2012 
Table 6. New (in bold and italics) and tying high counts for individual species 2011.

\begin{tabular}{|c|c|c|c|c|}
\hline LOCATION & $\begin{array}{c}2011 \\
\text { COUNT }\end{array}$ & SPECIES & $\begin{array}{l}\text { PREVIOUS } \\
\text { HIGH }\end{array}$ & LOCATION, YEAR \\
\hline Gardiner Dam & 50 & Cackling Goose & 33 & Gardiner Dam, 2005 \\
\hline Gardiner Dam & 27 & Redhead & 15 & Gardiner Dam, 1999 \\
\hline Estevan & 7 & $\begin{array}{l}\text { Ring-necked } \\
\text { Duck }\end{array}$ & 4 & Grayson, 2005 \\
\hline Gardiner Dam & 2 & $\begin{array}{l}\text { White-winged } \\
\text { Scoter }\end{array}$ & 2 & Cold River, 1974 \\
\hline Squaw Rapids & 1 & Long-tailed Duck & 1 & $\begin{array}{l}\text { Ft Qu'Appelle, } 1968 \\
\text { (count period); } \\
\text { Saskatoon, } 1997 \text {; } \\
\text { Gardiner Dam } 2002\end{array}$ \\
\hline $\begin{array}{l}\text { Squaw Rapids } \\
\text { (count period) }\end{array}$ & 1 & $\begin{array}{l}\text { Barrow's } \\
\text { Goldeneye }\end{array}$ & 1 & $\begin{array}{l}\text { Squaw Rapids, 1996; } \\
\text { Saskatoon } 2007 \text { (count } \\
\text { period) }\end{array}$ \\
\hline Estevan & 5 & $\begin{array}{l}\text { Hooded } \\
\text { Merganser }\end{array}$ & 3 & Estevan, 1960, 2010 \\
\hline Estevan & 2 & Pied-billed Grebe & 2 & Regina, 1955, 1973 \\
\hline Gardiner Dam & 1 & Horned Grebe & 1 & $\begin{array}{l}\text { Regina, 1959, 1960, } \\
\text { 1961, 1977; Saskatoon, } \\
\text { 1983; Coronach, } 1997\end{array}$ \\
\hline Gardiner Dam & 1 & $\begin{array}{l}\text { Red-necked } \\
\text { Grebe }\end{array}$ & 1 & $\begin{array}{l}\text { Regina, 1968; Squaw } \\
\text { Rapids } 1988\end{array}$ \\
\hline Coronach & 1 & Western Grebe & 1 & $\begin{array}{l}\text { Regina, 1956, 1969; } \\
\text { Gardine r D a m, } \\
\text { 1979, 1988, 1997; } \\
\text { Qu'Apppelle Dam, } \\
\text { 1990, 2005; Coronach, } \\
\text { 1998, } 1999\end{array}$ \\
\hline Regina & 45 & Snowy OwI & 36 & Regina 1997 \\
\hline $\begin{array}{l}\text { Prince Albert } \\
\text { N.P. }\end{array}$ & 142 & $\begin{array}{l}\text { Boreal } \\
\text { Chcikadee }\end{array}$ & 52 & $\begin{array}{l}\text { Prince Albert N.P., } \\
1987,1996\end{array}$ \\
\hline Ft. Qu'Appelle & 1 & Winter Wren & 1 & Ft Walsh, 1999 \\
\hline Togo & 1 & $\begin{array}{l}\text { Orange-crowned } \\
\text { Warbler }\end{array}$ & 1 & $\begin{array}{l}\text { Saskatoon, } 1997 \\
\text { (count Period) }\end{array}$ \\
\hline $\begin{array}{l}\text { Prince Albert } \\
\text { (count period) }\end{array}$ & 2 & Northern Cardinal & 2 & Kamsack, 1998 \\
\hline Indian Head & 2 & $\begin{array}{l}\text { Yellow-headed } \\
\text { Blackbird }\end{array}$ & 1 & $\begin{array}{l}\text { Moose Jaw, } 1995 \\
\text { (count period) }\end{array}$ \\
\hline
\end{tabular}


Table 7. Population changes in numbers of selected species in $2011^{*}$ compared to 2010 and the average for 5 years (2006-2010), based on the number of birds per party hour. A minus sign indicates a decrease from previous years.

\begin{tabular}{|c|c|c|c|c|c|}
\hline Species & 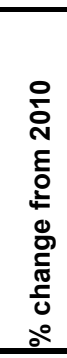 & 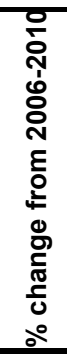 & & 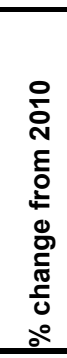 & 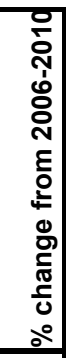 \\
\hline Canada Goose & 720 & 193 & Black-billed Magpie & 10 & 24 \\
\hline Mallard & 144 & 54 & Common Raven & 21 & 38 \\
\hline Common Goldeneye & 26 & 25 & Horned Lark & -79 & -90 \\
\hline Gray Partridge & -79 & -78 & Black-capped Chickadee & -9 & 0 \\
\hline Ring-necked Pheasant & -38 & -33 & Boreal Chickadee & 216 & 116 \\
\hline Ruffed Grouse & -23 & -21 & Red-breasted Nuthatch & -36 & -41 \\
\hline Sharp-tailed Grouse & -46 & -38 & White-breasted Nuthatch & -21 & -6 \\
\hline Bald Eagle & 15 & 93 & Brown Creeper & 24 & 33 \\
\hline Northern Goshawk & -19 & -31 & Golden-crowned Kinglet & -33 & 18 \\
\hline Golden Eagle & 52 & 52 & American Robin & 85 & -74 \\
\hline Merlin & 69 & 79 & European Starling & 244 & 261 \\
\hline Prairie Falcon & -22 & -35 & Bohemian Waxwing & -55 & -28 \\
\hline Rock Pigeon & 10 & 8 & Dark-eyed Junco & 69 & 37 \\
\hline Great Horned Owl & 21 & -1 & Snow Bunting & -4 & -45 \\
\hline Snowy Owl & 659 & 272 & Pine Grosbeak & 188 & 27 \\
\hline Short-eared Owl & 242 & -36 & House Finch & -12 & -11 \\
\hline Downy Woodpecker & -35 & -21 & White-winged Crossbill & -43 & -49 \\
\hline Hairy Woodpecker & -32 & -20 & Common Redpoll & 552 & 139 \\
\hline Northern Flicker & 81 & -10 & Hoary Redpoll & 47 & -11 \\
\hline Pileated Woodpecker & 8 & -3 & Pine Siskin & -28 & -75 \\
\hline Northern Shrike & -28 & -14 & Evening Grosbeak & 1 & -2 \\
\hline Gray Jay & 71 & 17 & House Sparrow & 5 & \\
\hline Blue Jay & -1 & 8 & & & \\
\hline
\end{tabular}

${ }^{*}$ As tremendous changes can occur in small sample sizes only those species recorded on an annual average of six or more counts are included. 